\title{
Chemical and Biological Research on Herbal Medicines Rich in Xanthones
}

\author{
Jingya Ruan ${ }^{1}$, Chang Zheng ${ }^{1}$, Yanxia Liu ${ }^{1}$, Lu Qu ${ }^{2}$, Haiyang Yu ${ }^{2}$, Lifeng Han ${ }^{2}$, Yi Zhang ${ }^{1,2, *}$ \\ and Tao Wang $1,2, *$ \\ 1 Tianjin State Key Laboratory of Modern Chinese Medicine, 312 Anshanxi Road, Nankai District, Tianjin \\ 300193, China; Ruanjy19930919@163.com (J.R.); 18702270347@163.com (C.Z.); liuyanxia210@163.com (Y.L.) \\ 2 Tianjin Key Laboratory of TCM Chemistry and Analysis, Institute of Traditional Chinese Medicine, Tianjin \\ University of Traditional Chinese Medicine, 312 Anshan Road, Nankai District, Tianjin 300193, China; \\ qululuhan88@163.com (L.Q.); yuhaiyang19830116@hotmail.com (H.Y.); hanlifeng_1@163.com (L.H.) \\ * Correspondence: zhwwxzh@263.net (Y.Z.); wangtao@tjutcm.edu.cn (T.W.); \\ Tel./Fax: +86-22-5959-6163 (Y.Z. \& T.W.)
}

Received: 11 September 2017; Accepted: 9 October 2017; Published: 11 October 2017

\begin{abstract}
Xanthones, as some of the most active components and widely distributed in various herb medicines, have drawn more and more attention in recent years. So far, 168 species of herbal plants belong to 58 genera, 24 families have been reported to contain xanthones. Among them, Calophyllum, Cratoxylum, Cudrania, Garcinia, Gentiana, Hypericum and Swertia genera are plant resources with great development prospect. This paper summarizes the plant resources, bioactivity and the structure-activity relationships (SARs) of xanthones from references published over the last few decades, which may be useful for new drug research and development on xanthones.
\end{abstract}

Keywords: herbal medicines; xanthones; plant sources; pharmacology; gambogic acid; structure-activity relationships

\section{Introdution}

Xanthones (IUPAC name $9 H$-xanthen-9-one) are a kind of phenolic acid with a three-ring skeleton, widely distributed in herbal medicines. These constituents display a vast range of bioactitivies, including anticancer, anti-oxidative, antimicrobial, antidiabetic, antiviral, and anti-inflammatory effects. So far, at least 515 natural xanthones from 20 families of higher plants (122 species in 44 genera) have been summarized in a few reviews [1-3]. These reviews were limited to xanthones with anticancer and anti-inflammatory activities [4]. Their structure-activity relationships (SARs) were also not mentioned.

Over the past few decades, xanthones have become an important resource for drug development. For example, gambogic acid, a prenyl xanthone isolated from Garcinia hanburyi (Clusiaceae), exhibited remarkable apoptosis, cell proliferation and tumor angiogenesis bioactivities, along with anti-oxidant, and anti-inflammatory activities [5,6] and synergistic anticancer activity [7,8]. A phase II clinical trial using gambogic acid in combination with anticancer drugs was carried out in China [9]. Besides gambogic acid mentioned above, mangosteen, another of the most well-known xanthones, has been used as a dietary supplement to improve immune function, decrease serum C-reactive protein levels and increase the ratio of $T$ helper cells [10].

Xanthones are mainly isolated from herbal medicines. Between 1988 and 2016, 168 species of herbal medicinal plant belonging to 58 genera, and 24 families were reported to contain xanthones. This review summarizes the phytochemistry, bioactivity and structure-activity relationships (SARs) of xanthones, which may be helpful to the further new drug research and development. 


\section{Plant Sources of Xanthones}

Table 1 summarizes the phytochemical research on xanthones found in 168 plant species belonging to 58 genera and 24 families.

Table 1. Plant distribution of xanthones.

\begin{tabular}{|c|c|c|c|}
\hline Family & Genus & Species & Reference \\
\hline Acanthaceae & Andrographis & A. paniculata (Burm. f.) Nees & [11] \\
\hline \multirow{2}{*}{ Anacardiaceae } & Mangifera & M. indica $\mathrm{L}$. & [12] \\
\hline & Rhus & R. coriaria $\mathrm{L}$. & [13] \\
\hline \multirow{2}{*}{ Annonaceae } & Anaxagorea & A. luzonensis A. Gray & [14] \\
\hline & Guatteria & G. blepharophylla Mart. & [15] \\
\hline Asparagus & Ledebouria & L. ovatifolia (Schrad.) Jessop & [16] \\
\hline \multirow{2}{*}{ Asparagaceae } & Anemarrhena & A. asphodeloides Bunge & [17] \\
\hline & Drimiopsis & D. maculate Lindl. \& Paxton & [18] \\
\hline Asteraceae & Santolina & S. insularis (Gennari ex Fiori) Arrigoni & [19] \\
\hline Bignoniaceae & Arrabidaea & A. samydoides (Cham.) Sandwith & [20] \\
\hline \multirow[t]{18}{*}{ Bombacaceae } & Bombax & B. ceiba L. & [21] \\
\hline & & A. floribunda Oliv. & [22] \\
\hline & Allanblackia & A. gabonensis (Pellegr.) Bamps & [23] \\
\hline & & A. monticola Staner L. C. & [24] \\
\hline & Bonnetia & B. stricta Mart. & [25] \\
\hline & & C. brasiliense Cambess. & [26] \\
\hline & & C. caledonicum Vieill. ex Planch. \& Triana & [27] \\
\hline & & C. decipiens Wight & [28] \\
\hline & & C. gracilipes Merr. & [29] \\
\hline & & C. inophyllum $\mathrm{L}$. & [30] \\
\hline & Calonhullum & C. membranaceum Gardner \& Champ. & [31] \\
\hline & Calopnyıum & C. panciflorum A. C. Smith & [32] \\
\hline & & C. pinetorum Bisse & [33] \\
\hline & & C. soulattri Burm. f. & [34] \\
\hline & & C. symingtonianum M.R. Hend. \& Wyatt-Sm. & [35] \\
\hline & & C. thorelii Pierre & [36] \\
\hline & & C. thwaitesii Planch. \& Triana & [37] \\
\hline & Chrysochlamys & C. tenuis Hammel & [38] \\
\hline \multirow{25}{*}{$\begin{array}{l}\text { Clusiaceae } \\
\text { (or Guttiferae) }\end{array}$} & Clusia & C. pernambucensis G. Mariz & [39] \\
\hline & Cratorulum & C. cochinchinensis (Lour.) Blume & [40] \\
\hline & Cratoxylum & C. formosum sp. Pruniflorum (Kurz) Gogelein & [41] \\
\hline & & G. afzelii Engl. & [42] \\
\hline & & G. bracteata C.Y. Wu ex Y.H. Li & [43] \\
\hline & & G. cambogia (Gaertn.) Desr. & [44] \\
\hline & & G. cantleyana Whitmore & [45] \\
\hline & & G. cowa Roxb. ex Choisy & [46] \\
\hline & & G. dioica Blume & [47] \\
\hline & & G. dulcis (Roxb.) Kurz & [48] \\
\hline & & G. eugenifolia Wall. ex T. Anderson & [49] \\
\hline & & G. fusca Pierre & [50] \\
\hline & & G. goudotiana (Planch. \& Triana) P. Sweeney \& Z.S. Rogers & [51] \\
\hline & Garcinia & G. griffthii T. Anderson & [52] \\
\hline & & G. hanburyi Hook. f. & [53] \\
\hline & & G. hombroniana Pierre & [54] \\
\hline & & G. lancilimba C.Y. Wu ex Y.H. Li & [55] \\
\hline & & G. lateriflora Blume & [56] \\
\hline & & G. linii C.E. Chang & [57] \\
\hline & & G. mangostana L. & [58] \\
\hline & & G. merguensis Wight & [59] \\
\hline & & G. multiflora Champ. ex Benth. & [60] \\
\hline & & G. nigrolineata Planch. ex T. Anderson & [61] \\
\hline & & G. nitida Pierre & [62] \\
\hline & & G. nobilis Engl. & [63] \\
\hline
\end{tabular}


Table 1. Cont.

\begin{tabular}{|c|c|c|c|}
\hline Family & Genus & Species & Reference \\
\hline \multirow{35}{*}{$\begin{array}{l}\text { Clusiaceae } \\
\text { (or Guttiferae) }\end{array}$} & \multirow{23}{*}{ Garcinia } & G. nujiangensis C.Y. Wu \& Y.H. Li & [64] \\
\hline & & G. oblongifolia Champ. ex Benth. & [65] \\
\hline & & G. oligantha Merr. & [66] \\
\hline & & G. oliveri Pierre & [67] \\
\hline & & G. parvifolia (Miq.) Miq. & [68] \\
\hline & & G. paucinervis Chun \& F.C. How & [69] \\
\hline & & G. pedunculata Roxb. ex Buch.-Ham. & [70] \\
\hline & & G. penangiana Pierre & [71] \\
\hline & & G. polyantha Oliv. & [72] \\
\hline & & G. porrecta Laness. & [68] \\
\hline & & G. propinqua Craib & [73] \\
\hline & & G. rigida Miq. & [74] \\
\hline & & G. schomburgkiana Pierre & [75] \\
\hline & & G. scortechinii King & [76] \\
\hline & & G. smeathmannii (Planch. \& Triana) Oliv. & [77] \\
\hline & & G. staudtii Engl. & [78] \\
\hline & & G. subelliptica Merr. & [79] \\
\hline & & G. succifolia Kurz & [80] \\
\hline & & G. tetralata C.Y. Wu ex Y.H. Li & [81] \\
\hline & & G. vieillardii Pierre & [82] \\
\hline & & G. virgate Vieill. & [83] \\
\hline & & G. xanthochymus Hook. f. ex T. Anderson & {$[84]$} \\
\hline & & G. xipshuanbannaensis Y.H. Li & [85] \\
\hline & \multirow{2}{*}{ Kielmeyera } & K. coriacea Mart. & [86] \\
\hline & & K. variabilis Mart. \& Zucc. & [87] \\
\hline & Mammea & M. siamensis T. Anderson & [88] \\
\hline & \multirow[b]{2}{*}{ Mesua } & M. ferrea $\mathrm{L}$. & [89] \\
\hline & & M. hexapetala (Hook. f.) P.S. Ashton & {$[90]$} \\
\hline & \multirow{3}{*}{ Psorospermum } & P. adamauense Engl. & [91] \\
\hline & & P. febrifugum Spach & [92] \\
\hline & & P. molluscum (Pers.) Hochr. & [93] \\
\hline & Rheedia & R. acuminata (Ruiz \& Pav.) Planch. \& Triana & [94] \\
\hline & Symphonia & S. globulifera L.f. & [95] \\
\hline & & V. laurentii De Wild. & [96] \\
\hline & Vismia & V. rubescens Oliv. & [96] \\
\hline \multirow{4}{*}{ Fabaceae } & Caesalpinia & C. sappan $\mathrm{L}$. & [97] \\
\hline & Cassia & C. obtusifolia L. & [98] \\
\hline & Cyclopia & C. genistoides (L.) Vent. & [99] \\
\hline & Desmodium & D. caudatum (Thunb.) DC. & [100] \\
\hline Ganodermataceae & Gyrophora & G. proboscidea (L.) Ach. & [101] \\
\hline \multirow{18}{*}{ Gentianaceae } & Centaurium & C. spicatum (L.) Fritsch & [102] \\
\hline & \multirow{2}{*}{ Comastoma } & C. pedunculatum (Royle ex G. Don) Holub & [103] \\
\hline & & C. pulmonarium (Turcz.) Toyok. & [104] \\
\hline & \multirow{6}{*}{ Gentiana } & G. dinarica Beck & [105] \\
\hline & & G. kochiana Perr. \& Songeon & [106] \\
\hline & & G. lutea L. & [107] \\
\hline & & G. tizuensis Franch. & [108] \\
\hline & & G. utriculosa L. & {$[109]$} \\
\hline & & Gentiana dinarica Beck. & [110] \\
\hline & \multirow{3}{*}{ Gentianella } & G. acuta (Michx.) Hiitonen & [111] \\
\hline & & G. amarella (L.) Harry Sm. & [112] \\
\hline & & G. turkestanorum (Gand.) Holub & [113] \\
\hline & \multirow{2}{*}{ Gentianopsis } & G. barbata (Froel.) Ma & [114] \\
\hline & & G. paludosa (Hook. f.) Ma & [115] \\
\hline & \multirow[b]{2}{*}{ Halenia } & H. corniculata (L.) Cornaz & [116] \\
\hline & & H. elliptica D. Don & [117] \\
\hline & Lomatogonium & L. carinthiacum (Wulfen) A. Braun & [118] \\
\hline & Schultesia & S. lisianthoides (Griseb.) Benth. \& Hook. f. ex Hemsl. & [119] \\
\hline
\end{tabular}


Table 1. Cont.

\begin{tabular}{|c|c|c|c|}
\hline Family & Genus & Species & Reference \\
\hline \multirow{15}{*}{ Gentianaceae } & \multirow{14}{*}{ Swertia } & S. chirayita (Roxb.) H. Karsten & [120] \\
\hline & & S. cordata (Wall. ex G. Don) C.B. Clarke & [121] \\
\hline & & S. corymbosa Wight ex Griseb. & [122] \\
\hline & & S. cuneata Wall. ex D. Don & [123] \\
\hline & & S. elata Harry Sm. & [124] \\
\hline & & S. franchetiana Harry Sm. & [125] \\
\hline & & S. kouitchensis Franch. & [126] \\
\hline & & S. longifolia Boiss. & [127] \\
\hline & & S. minor (Griscb.) Knobl. & [128] \\
\hline & & S. mussotii Franch. & [129] \\
\hline & & S. paniculata & [130] \\
\hline & & S. pseudochinensis H. Hara & [131] \\
\hline & & S. punicea Hemsl. & [132] \\
\hline & & S. speciosa Wall. & [133] \\
\hline & Tachia & T. grandiflora Maguire \& Weaver & [134] \\
\hline \multirow{2}{*}{ Hippocrateaceae } & \multirow{2}{*}{ Salacia } & S. chinensis L. & [135] \\
\hline & & S. elliptica (Mart.) G. Don & [136] \\
\hline Hyacinthaceae & Scilla & S. scilloides (Lindl.) Druce & [137] \\
\hline \multirow{11}{*}{ Hypericaceae } & \multirow{11}{*}{ Hypericum } & H. ascyron L. & [138] \\
\hline & & H. attenuatum Fisch. ex Choisy & [139] \\
\hline & & H. chinense $\mathrm{L}$. & [140] \\
\hline & & H. erectum Thunb. & [141] \\
\hline & & H. lanceolatum Lam. & [142] \\
\hline & & H. oblongifolium Choisy & [143] \\
\hline & & H. patulum Thunb. & [144] \\
\hline & & H. perforatum L. & [145] \\
\hline & & H. sampsonii Hance & [146] \\
\hline & & H. scabrum L. & [147] \\
\hline & & H. styphelioides A. Rich. & [148] \\
\hline Iridaceae & Iris & I. nigricans Dinsm. & [149] \\
\hline \multirow{2}{*}{ Loganiaceae } & \multirow{2}{*}{ Anthocleista } & A. schweinfurthii Gilg & [150] \\
\hline & & A. vogelii Planch. & [151] \\
\hline \multirow{7}{*}{ Moraceae } & \multirow{3}{*}{ Artocarpus } & A. kemando Miq. & [152] \\
\hline & & A. nobilis Thwaites & [153] \\
\hline & & A. obtusus F.M. Jarrett & [154] \\
\hline & \multirow{3}{*}{ Cudrania } & C. cochinchinensis (Lour.) Yakuro Kudo \& Masam. & [155] \\
\hline & & C. fruticosa (Roxb.) Wight ex Kurz & [156] \\
\hline & & C. tricuspidata (Carrière) Bureau ex Lavallée & [157] \\
\hline & Maclura & M. cochinchinensis (Lour.) Corner & [158] \\
\hline Onagraceae & Oenothera & O. biennis L. & [159] \\
\hline Parmeliaceae & Usnea & U. hirta (L.) Weber ex F.H. Wigg & [160] \\
\hline \multirow{12}{*}{ Polygalaceae } & Bredemeyera & B. floribunda Willd. & [161] \\
\hline & Moutabea & M. guianensis Aubl. & [162] \\
\hline & \multirow{3}{*}{ Polygala } & P. caudata Rehder \& E.H. Wilson & [163] \\
\hline & & P. crotalarioides Buch.-Ham. ex DC. & [164] \\
\hline & & P. cyparissias A. St.-Hil. \& Moq. & [165] \\
\hline & \multirow{7}{*}{ Securidaca } & P. hongkongensis Hemsl. & [166] \\
\hline & & P. japonica Houtt. & [167] \\
\hline & & P. karensium Kurz & [168] \\
\hline & & P. tenuifolia Willd. & [169] \\
\hline & & P. wattersii Hance & [170] \\
\hline & & S. inappendiculata Hassk. & [171] \\
\hline & & S. longepedunculata Fresen. & [172] \\
\hline \multirow{2}{*}{ Rubiaceae } & Coffea & C. pseudozanguebariae Bridson & [173] \\
\hline & Morinda & M. citrifolia $\mathrm{L}$. & [174] \\
\hline Theaceae & Pentadesma & P. butyrace Sabine & [175] \\
\hline Xanthorrhoeaceae & Bulbine & B. frutescens (L.) Willd. & [176] \\
\hline Zingiberaceae & Hedychium & H. gardnerianum Sheppard ex Ker Gawl. & [177] \\
\hline
\end{tabular}


Among them, the Calophyllaceae, Gentianaceae and Guttiferae are the most widely distributed families.

\section{Bioactivities of Xanthones}

Recently, some xanthones have been reported to be useful in the treatment of cancer, oxidation, microbial infection, diabetes, inflammation, virus infection et al. Target-based and structure-based activity evaluation has revealed that xanthones are good source of medicine for the treatment of various type of disease. In this part, we summarized pharmacological activities and SARs result of xanthones.

\subsection{Effects on Cytotoxicity and Proliferation}

Cancer cytotoxicities of xanthones against leukemia cell lines were evaluated, which were isolated from Artocarpus [154], Calophyllum [34], Garcinia [178], Hypericum [140] genera herbal medicines. On the other hand, the xanthones phylattrin (1), caloxanthone C (2), brasixanthone B (3), macluraxanthone (4), and soulattrin (5) (Figure 1) obtained from C. soulattri [34] showed cytotoxic activities against the chronic myelogenous leukemia cell line (K562) $\left(\mathrm{IC}_{50}: 22.10 \pm 0.61,18.20 \pm 0.76,31.00 \pm 0.21\right.$, $5.28 \pm 0.22$, and $2.23 \pm 0.13 \mu \mathrm{M}$, respectively). Their MTT test results indicated that with increasing number of hydroxyl groups, the anti-proliferative activity was enhanced $(2<4)$.
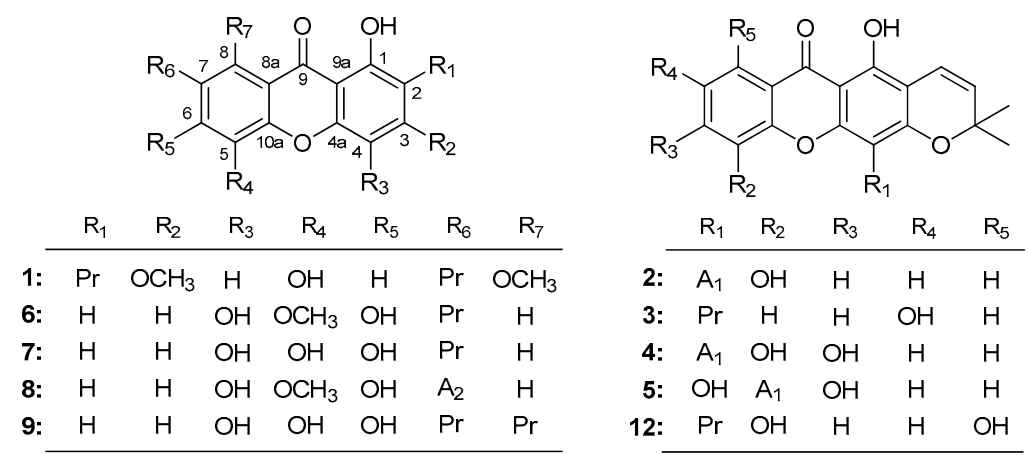

\begin{tabular}{rccccc} 
& $\mathrm{R}_{1}$ & $\mathrm{R}_{2}$ & $\mathrm{R}_{3}$ & $\mathrm{R}_{4}$ & $\mathrm{R}_{5}$ \\
\cline { 2 - 6 } 2: & $\mathrm{A}_{1}$ & $\mathrm{OH}$ & $\mathrm{H}$ & $\mathrm{H}$ & $\mathrm{H}$ \\
3: & $\mathrm{Pr}$ & $\mathrm{H}$ & $\mathrm{H}$ & $\mathrm{OH}$ & $\mathrm{H}$ \\
4: & $\mathrm{A}_{1}$ & $\mathrm{OH}$ & $\mathrm{OH}$ & $\mathrm{H}$ & $\mathrm{H}$ \\
5: & $\mathrm{OH}$ & $\mathrm{A}_{1}$ & $\mathrm{OH}$ & $\mathrm{H}$ & $\mathrm{H}$ \\
12: & $\mathrm{Pr}$ & $\mathrm{OH}$ & $\mathrm{H}$ & $\mathrm{H}$ & $\mathrm{OH}$ \\
\hline
\end{tabular}<smiles>COc1c2c(cc3c(=O)c4c(O)ccc(O)c4oc13)C(O)[C@H](C(C)(C)O)O2</smiles>

10<smiles>COc1c2c(cc3c(=O)c4c(O)ccc(O)c4oc13)CC(C(C)(C)O)O2</smiles>

11

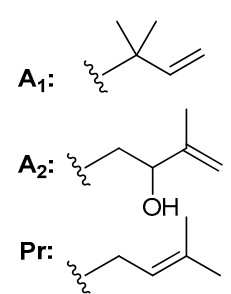

Figure 1. The structures of compounds 1-12.

In addition, the tests carried out by Niu et al. [43] supplemented the conclusions mentioned above. Thirty-one kinds of xanthones, including 1,4,6-trihydroxy-5-methoxy-7-prenylxanthone (6), 1,4,5,6-tetrahydroxy-7-prenylxanthone (7), bracteaxanthone III (8), 1,4,5,6-tetrahydroxy-7,8-di (3-methylbut-2-enyl)xanthone (9), bracteaxanthones V (10), IV (11), and garcinexanthone B (12) (Figure 1) were obtained from G. bracteata. Activity screening results revealed that the isoprenyl group played an important role in the HL-60 cytotoxicity. Among them, 7 and $\mathbf{9}$ showed stronger inhibitory abilities with $\mathrm{IC}_{50}$ at $10.1 \pm 3.1,2.8 \pm 1.1 \mu \mathrm{M}$, respectively. The activities difference between these compounds suggested that along with the number of isoprenyl group increasing, the cytotoxicities became stronger. Meanwhile, the hydroxylation $(6>8)$ or the cyclization into a furan or pyran ring of isoprenyl group $(\mathbf{6}>\mathbf{1 0}-\mathbf{1 2})$ lowered the activity compared with corresponding compounds $\left(\mathrm{IC}_{50}: 9.9 \pm 0.8,21.0 \pm 0.5,22.2 \pm 0.6,18.0 \pm 0.7\right.$ and $22.8 \pm 0.4 \mu \mathrm{M}$ for $\mathbf{6 ,}, \mathbf{8}, \mathbf{1 0}, \mathbf{1 1}$ and 12, respectively).

Xanthones from Garcinia [66], Polygala [166] genera plants were found to exhibit inhibitory activities in A549 lung cancer cell line. Oliganthins H (13), I (14), gaudichaudione H (15), cantleyanone 
(16), and oliganthone B (17) (Figure 2) isolated from G. oligantha [66] showed anti-proliferative potency for $\mathrm{A} 549$ with $\mathrm{IC}_{50}$ at $5.0 \pm 0.32,5.5 \pm 0.47,3.0 \pm 0.49,2.9 \pm 0.42,3.9 \pm 0.86 \mu \mathrm{M}$, respectively. Compounds 15, 16 and 17 exhibited stronger inhibitory abilities compared with 13 and 14, which indicated that caged-xanthones may have better performances on inhibiting the growth of A549 cell line. A cytotoxicity screen on desoxygambogenin (18), isogambogenic acid (19), and $10 \alpha$-ethoxy-9,10-dihydrogambogenic acid (20) (Figure 2) from G. hanburyi [53] against the A549 cell line indicated that the carboxylation of the side chain of the caged-xanthones decreased the activity (18 > 19), and the caged-xanthones with an olefinic bond between C-9 and C-10 depslayed higher inhibitory ability than hydroxyl substituted ones $(\mathbf{1 9}>\mathbf{2 0})$.
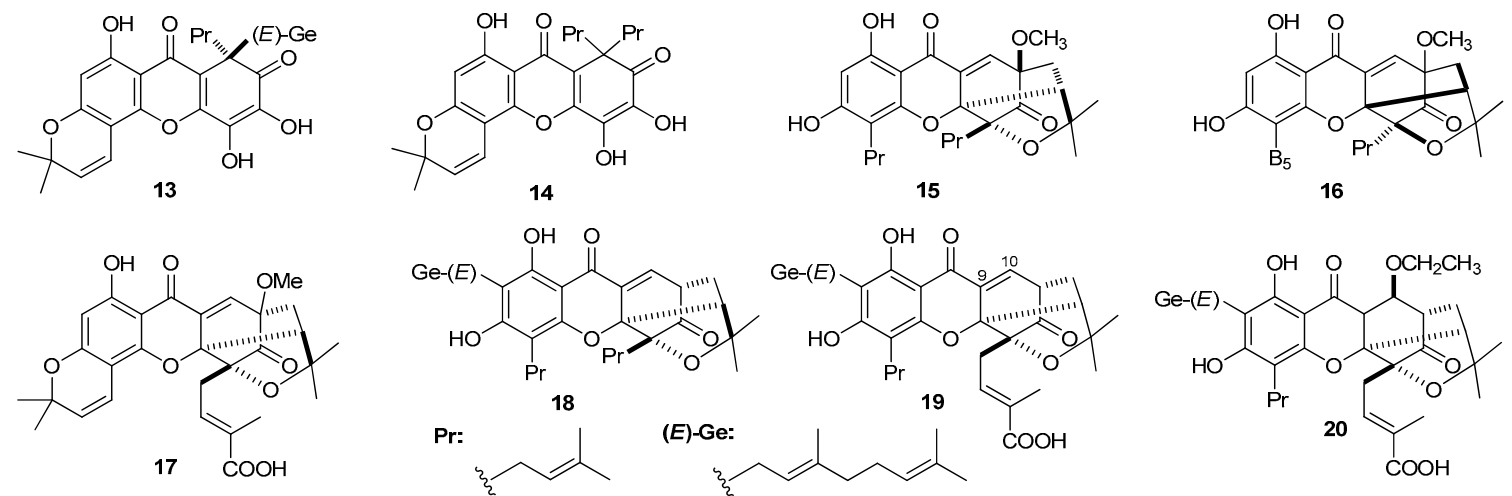

Figure 2. The structures of compounds 13-20.

Xanthones isolated from Garcinia genus plants showed significant inhibitory effect in colon cancer cells. The cytotoxicity of cowaxanthone (21), rubraxanthone (22), $\alpha$-mangostin (23), cowanin (24), cowanol (25) (Figure 3) from G. oliveri [67] against DLD-1 cell line was confirmed by the MTT method with $\mathrm{IC}_{50}$ value at $24.4 \pm 0.7,33.9 \pm 2.2,12.2 \pm 0.4,13.2 \pm 0.2,14.8 \pm 2.1 \mu \mathrm{M}$, respectively. SARs results suggested that free 3,6-dihydroxyl group and isoprenyl side chain at C-2 and C-8 of were active units.<smiles>[R]c1cc2oc3c([R9])c([R])c([R])c(O)c3c(=O)c2c([R])c1[R]</smiles>

\begin{tabular}{lcccccc} 
& $\mathrm{R}_{1}$ & $\mathrm{R}_{2}$ & $\mathrm{R}_{3}$ & $\mathrm{R}_{4}$ & $\mathrm{R}_{5}$ & $\mathrm{R}_{6}$ \\
\cline { 2 - 7 } 21: & $(E)-\mathrm{Ge}$ & $\mathrm{OH}$ & $\mathrm{H}$ & $\mathrm{OH}$ & $\mathrm{OCH}_{3}$ & $\mathrm{H}$ \\
22: & $\mathrm{H}$ & $\mathrm{OH}$ & $\mathrm{H}$ & $\mathrm{OH}$ & $\mathrm{OCH}_{3}$ & $(E)-\mathrm{Ge}$ \\
23: & $\mathrm{Pr}$ & $\mathrm{OH}$ & $\mathrm{H}$ & $\mathrm{OH}$ & $\mathrm{OCH}_{3}$ & $\mathrm{Pr}$ \\
24: & $\mathrm{Pr}$ & $\mathrm{OH}$ & $\mathrm{H}$ & $\mathrm{OH}$ & $\mathrm{OCH}_{3}$ & $(E)-\mathrm{Ge}$ \\
25: & $\mathrm{A}_{3}$ & $\mathrm{OH}$ & $\mathrm{H}$ & $\mathrm{OH}$ & $\mathrm{OCH}_{3}$ & $(E)-\mathrm{Ge}$ \\
26: & $\mathrm{Pr}$ & $\mathrm{OH}$ & $\mathrm{H}$ & $\mathrm{OH}$ & $\mathrm{OCH}_{3}$ & $\mathrm{~A}_{4}$ \\
27: & $\mathrm{Pr}$ & $\mathrm{OCH}_{3}$ & $\mathrm{H}$ & $\mathrm{OH}$ & $\mathrm{OCH}_{3}$ & $\mathrm{Pr}$ \\
28: & $\mathrm{Pr}$ & $\mathrm{OCH}$ & $\mathrm{H}$ & $\mathrm{OCH}$ & $\mathrm{OCH}_{3}$ & $\mathrm{Pr}$ \\
29: & $\mathrm{A}_{3}$ & $\mathrm{OH}$ & $\mathrm{H}$ & $\mathrm{OH}$ & $\mathrm{OCH}_{3}$ & $\mathrm{~A}_{5}$ \\
30: & $\mathrm{A}_{3}$ & $\mathrm{OH}$ & $\mathrm{H}$ & $\mathrm{OH}$ & $\mathrm{OCH}_{3}$ & $\mathrm{~A}_{6}$ \\
\cline { 2 - 6 } & & & & & &
\end{tabular}

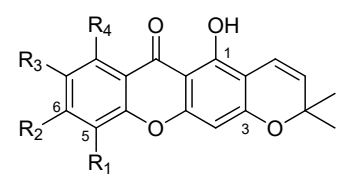

\begin{tabular}{lcccc} 
& $\mathrm{R}_{1}$ & $\mathrm{R}_{2}$ & $\mathrm{R}_{3}$ & $\mathrm{R}_{4}$ \\
\cline { 2 - 5 } 31: & $\mathrm{OH}$ & $\mathrm{OH}$ & $\mathrm{H}$ & $\mathrm{H}$ \\
32: & $\mathrm{H}$ & $\mathrm{OH}$ & $\mathrm{OCH}_{3}$ & $(E)-\mathrm{Ge}$ \\
34: $\mathrm{OCH}_{3}$ & $\mathrm{OCH}_{3}$ & $\mathrm{OCH}_{3}$ & $\mathrm{OCH}_{3}$ \\
35: $\mathrm{OCH}_{3}$ & $\mathrm{OH}$ & $\mathrm{OCH}_{3}$ & $\mathrm{OCH}_{3}$ \\
36: $\mathrm{OCH}_{3}$ & $\mathrm{OH}$ & $\mathrm{OCH}_{3}$ & $\mathrm{H}$ \\
\hline
\end{tabular}

Pr:<smiles>CCCC=C(C)C</smiles>

(E)-Ge:<smiles>CCCC=C(C)CCC=C(C)C</smiles><smiles>COc1c(O)cc2oc3cc(O)c4c(c3c(=O)c2c1CC=C(C)C)OC(C)(C)CC4</smiles><smiles>CCCCC(C)(C)O</smiles>

$A_{5}$ :<smiles>CCCCC(C)(O)CCC=C(C)C</smiles><smiles>CC/C=C(\C)CCCC(C)(C)O</smiles>

Figure 3. The structures of compounds 21-36.

$\alpha$-Mangostin (23), cowanin (24), cowanol (25), garcinone D (26), $\beta$-mangostin (27), fuscaxanthone C (28), fuscaxanthone I (29), kaennacowanol A (30), jacareubin (31), fuscaxanthone A (32), and 1-isomagostin (33) (Figure 3) were obtained from G. cowa [179]. To cervical cancer Hela cell line, $\mathrm{IC}_{50}$ value of cytotoxicities were $13.69,11.68,12.19,22.58,12.78$, inactive, 17.20, 16.70, 11.43, inactive, $34.04 \mu \mathrm{M}$, respectively. SARs analysis results indicated that geranyl moiety at C-8 (24, 25 > 23; 29, 
$30>26)$ and the hydroxyl group at C-1, C-3, C-5 and C-6 enhanced their cytotoxicities $(23>33,24>32$, $31>32,27>28$ ).

Cylindroxanthones A-C (34, 35 and 36) (Figure 3) were gained from G. cylindrocarpa [180], along with the increase of methoxy, the anti-proliferative potency against oral epidermoid $\mathrm{KB}$ cell line $\left(\mathrm{IC}_{50}\right.$ : $2.36,59.05$ and $57.24 \mu \mathrm{M}$ for 34,35 and 36 respectively) increased. The results indicated that the oxidation of the unsaturated isoprenyl group reduced anti-proliferative activity.

Neuroblastoma (SHSY5Y) cell line proliferation could be inhibited by neriifolone A (37), cudraxanthones A (38), L (39), cudratrixanthones C (40), G (41), H (42), I (43), O (44), 3-O-methylcudratrixanthone $G$ (45), gerontoxanthone $C$ (46), 6-deoxyisojacareubin (47), and nigrolineaxanthone $F$ (48) (Figure 4) [181]. Moreover, gentiakochianin (49) and gentiacaulein (50) from G. kochiana [106] were also proved to promote cell cycle arrest in G2/M and G0/G1 phases in U251 human glioma cell line. Muchimangin B (51) [172] and allanxanthone A (52) (Figure 4) [23] inhibited the growth of pancreatic cancer (PANC-1), multiple myeloma (RPMI8226) and gastric cancer (BGC-823) cell lines. The SARs of above xanthones were not discussed for limitation on test sample number.

Although some xanthones showed significant inhibitory effects on cancer cell growth in vitro, the in vivo validatation report is rare, which limited their potential for development into new drug for anticancer.<smiles>[R]c1c([R])c([R])c2c(=O)c3c([R])c([R])c([R])c([R])c3oc2c1[R]</smiles>

\begin{tabular}{ccccccccc} 
& $\mathrm{R}_{1}$ & $\mathrm{R}_{2}$ & $\mathrm{R}_{3}$ & $\mathrm{R}_{4}$ & $\mathrm{R}_{5}$ & $\mathrm{R}_{6}$ & $\mathrm{R}_{7}$ & $\mathrm{R}_{8}$ \\
\cline { 2 - 8 } 37: & $\mathrm{OH}$ & $\mathrm{H}$ & $\mathrm{OH}$ & $\mathrm{A}_{1}$ & $\mathrm{OCH}_{3}$ & $\mathrm{OH}$ & $\mathrm{H}$ & $\mathrm{H}$ \\
40: & $\mathrm{OH}$ & $\mathrm{A}_{1}$ & $\mathrm{OH}$ & $\mathrm{H}$ & $\mathrm{Pr}$ & $\mathrm{OH}$ & $\mathrm{OH}$ & $\mathrm{H}$ \\
41: & $\mathrm{OH}$ & $\mathrm{H}$ & $\mathrm{OH}$ & $\mathrm{A}_{1}$ & $\mathrm{H}$ & $\mathrm{OH}$ & $\mathrm{OCH}_{3}$ & $\mathrm{~A}_{7}$ \\
45: & $\mathrm{OH}$ & $\mathrm{H}$ & $\mathrm{OCH}_{3}$ & $\mathrm{~A}_{1}$ & $\mathrm{H}$ & $\mathrm{OH}$ & $\mathrm{OH}$ & $\mathrm{A}_{2}$ \\
49: & $\mathrm{OH}$ & $\mathrm{H}$ & $\mathrm{OCH}_{3}$ & $\mathrm{H}$ & $\mathrm{H}$ & $\mathrm{H}$ & $\mathrm{OH}$ & $\mathrm{AH}_{2}$ \\
50: & $\mathrm{OH}$ & $\mathrm{H}$ & $\mathrm{OCH}_{3}$ & $\mathrm{H}$ & $\mathrm{H}$ & $\mathrm{H}$ & $\mathrm{OH}$ & $\mathrm{OCH}_{3}$ \\
52: & $\mathrm{OH}$ & $\mathrm{Pr}$ & $\mathrm{OH}$ & $\mathrm{A}_{1}$ & $\mathrm{OH}$ & $\mathrm{H}$ & $\mathrm{H}$ & $\mathrm{H}$ \\
\end{tabular}

$A_{1}:$<smiles>C=CC(C)(C)C</smiles>

$A_{2}:$<smiles>C=C(C)C(O)CCC</smiles>

Pr:<smiles>CCCC=C(C)C</smiles>

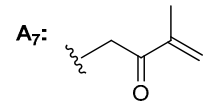<smiles>CC1(C)C=Cc2c(cc(O)c3c(=O)c4c5c(ccc4oc23)OC(C)(C)C=C5)O1</smiles>

38<smiles>CC1Oc2cc3oc4c(c(=O)c3c(O)c2C1(C)C)CC(O)C(C)(C)O4</smiles><smiles>CC(C)=CCc1c2c(c(O)c3c(=O)c4ccc(O)c(O)c4oc13)C(C)(C)C(C)O2</smiles>

46<smiles>CC1(C)C=Cc2c(cc(O)c3c(=O)c4cc(O)ccc4oc23)O1</smiles>

48<smiles>CCc1c(O)c(O)cc2c(=O)c3c4c(c(O)cc3oc12)C(C)(C)C(C)O4</smiles>

42<smiles></smiles><smiles>CC1(C)C=Cc2c(cc(O)c3c(=O)c4cccc(O)c4oc23)O1</smiles><smiles>COc1cc(OC)c(C(c2ccccc2)c2c(O)c(OC)c(O)c3c(=O)c4c(O)c(OC)c(O)cc4oc23)cc1OC</smiles>

Figure 4. The structures of compounds 37-52.

\subsection{Free Radical Scavenging Activity}

Free radicals are defined as atoms with one unpaired electron, which can be formed through natural physiological processes. Overproduction of free radicals can accelerate the progression of cancer, cardiovascular disease, and age-related diseases.

Symphoxanthone (53), subeliptenone B (54), garciniaxanthone E (55), garcinenone D (56), 1,3,5,6-tetrahydroxy-4,7,8-tri(3-methyl-2-butenyl)xanthone (57), garcinenone E (58) (Figure 5) were isolated from G. xanthochymus [84]. Their $\mathrm{IC}_{50}$ values against 1,1-diphenyl-2-picrylhydrazyl (DPPH) radical were $6.4,6.0,10.1,6.8,10.1,8.5 \mu \mathrm{M}$, respectively. SARs analysis indicated that the 
radical-scavenging activity was partly related to phenolic hydroxy moiety numbers. Within them, compounds with ortho diphenolic hydroxy groups showed significant radical-scavenging activity.

As described above, 1,8-dihydroxy-4,6-dimethoxyxanthone (59), 1,8-dihydroxy-4,6,7trimethoxyxanthone (60), 1,8-dihydroxy-4,5,6-trimetoxyxanthone (61), 1,8-dihydroxy-4,5,6,7-tetramethoxyxanthone (62), 1-hydroxy-4,5,6,7,8-pentamethoxyxanthone (63) (Figure 5) [162]. The DPPH scavenging order was $59(1.3 \mu \mathrm{g})<\mathbf{6 3}(0.6 \mu \mathrm{g})<\mathbf{6 0}$ and $\mathbf{6 1}(0.3 \mu \mathrm{g})<\mathbf{6 2}(0.15 \mu \mathrm{g})$. Agreeing with literature reports, the results indicated that DPPH radical scavenging activities might be attributed to the phenol-like $\mathrm{OH}$ groups at the xanthone skeleton [182].

\begin{tabular}{|c|c|c|c|c|c|c|c|c|}
\hline & $\mathrm{R}_{1}$ & $\mathrm{R}_{2}$ & $\mathrm{R}_{3}$ & $\mathrm{R}_{4}$ & $\mathrm{R}_{5}$ & $\mathrm{R}_{6}$ & $\mathrm{R}_{7}$ & $\mathrm{R}_{8}$ \\
\hline 53: & $\mathrm{OH}$ & $\mathrm{OH}$ & $\mathrm{H}$ & $A_{1}$ & $\mathrm{OH}$ & $\mathrm{OH}$ & $\mathrm{H}$ & $\mathrm{H}$ \\
\hline 54: & $\mathrm{OH}$ & $\mathrm{OH}$ & H & $A_{1}$ & $\mathrm{OH}$ & $\mathrm{OH}$ & $\mathrm{Pr}$ & $\mathrm{H}$ \\
\hline 55: & $\mathrm{OH}$ & $\mathrm{H}$ & $\mathrm{OH}$ & $\mathrm{H}$ & $\mathrm{OH}$ & $\mathrm{OH}$ & $(E)-G e$ & $\mathrm{Pr}$ \\
\hline 56: & $\mathrm{OH}$ & $\mathrm{H}$ & $\mathrm{OH}$ & $\mathrm{H}$ & $\mathrm{OH}$ & $\mathrm{OH}$ & $(E)-G e$ & $A_{4}$ \\
\hline 57: & $\mathrm{OH}$ & $\mathrm{H}$ & $\mathrm{OH}$ & $\mathrm{Pr}$ & $\mathrm{OH}$ & $\mathrm{OH}$ & $\mathrm{Pr}$ & $\mathrm{Pr}$ \\
\hline 58: & $\mathrm{OH}$ & $\mathrm{H}$ & $\mathrm{OH}$ & $\operatorname{Pr}$ & $\mathrm{OH}$ & $\mathrm{OH}$ & $\operatorname{Pr}$ & $\mathrm{A}_{4}$ \\
\hline 59: & $\mathrm{OH}$ & $\mathrm{H}$ & $\mathrm{OCH}_{3}$ & $\mathrm{H}$ & $\mathrm{OCH}_{3}$ & $\mathrm{H}$ & $\mathrm{H}$ & $\mathrm{OH}$ \\
\hline 60: & $\mathrm{OH}$ & $\mathrm{OCH}_{3}$ & $\mathrm{OCH}_{3}$ & $\mathrm{H}$ & $\mathrm{OCH}_{3}$ & $\mathrm{H}$ & $\mathrm{H}$ & $\mathrm{OH}$ \\
\hline 61: & $\mathrm{OH}$ & $\mathrm{H}$ & $\mathrm{OCH}_{3}$ & $\mathrm{OCH}_{3}$ & $\mathrm{OCH}_{3}$ & $\mathrm{H}$ & $\mathrm{H}$ & $\mathrm{OH}$ \\
\hline 62: & $\mathrm{OH}$ & $\mathrm{OCH}_{3}$ & $\mathrm{OCH}_{3}$ & $\mathrm{OCH}_{3}$ & $\mathrm{OCH}_{3}$ & $\mathrm{H}$ & $\mathrm{H}$ & $\mathrm{OH}$ \\
\hline 63: & $\mathrm{OCH}_{3}$ & $\mathrm{OCH}_{3}$ & $\mathrm{OCH}_{3}$ & $\mathrm{OCH}_{3}$ & $\mathrm{OCH}_{3}$ & $\mathrm{H}$ & $\mathrm{H}$ & $\mathrm{OH}$ \\
\hline
\end{tabular}

Figure 5. The structures of compounds 53-63.

\subsection{Anti-Microbial Activity}

Xanthones show suppressive effects on microorganisms, such as Gram-positive or negative bacteria and fungi. The resources include Allanblackia [23], Cassia [102], Centaurium [106], Cratoxylum [40], Garcinia [183], Hypericum [97], Kielmeyera [87], Psorospermum [91], Swertia [133], Usnea [160], and Vismia [96] genera plants.

2-Hydroxy-1-methoxyxanthone (64), 3-hydroxy-2-methoxyxanthone (65), 3,5-dihydroxy-4methoxyxanthone (66), 3,4-dihydroxy-2-methoxyxanthone (67), 5-hydroxy-1,3-dimethoxyxanthone (68), 4-hydroxy-2,3-dimethoxyxanthone (69), 3,4-dihydroxy-6,8-dimethoxyxanthone (70), 3,6-dihydroxy-1,4,8-trimethoxyxanthone (71), and kielcorin (72) (Figure 6) obtained from K. variabilis [87] showed strong activities against EMRSA-16. According to the results, phenol-like $\mathrm{OH}$ groups at the xanthone skeleton may play an important role in the inhibitory ability on the proliferation of microorganisms (MIC: 32, 32, 32, 16, 64, 64, > $512 \mathrm{mg} / \mathrm{L}$ for 64, 65, $66+70+71,67,68,69,72$, respectively). The isolates 1,7,8-trihydroxy-3-methoxyxanthone (73), gentiacaulein (74), and decussatin (75) (Figure 6) obtained from S. mussotii [133] were proved to inhibit the growth of $M$. tuberculosis with the same MICs at $125 \mu \mathrm{g} / \mathrm{mL}$, while 1,8-dihydroxy-2,6-dimethoxyxanthone (76) exhibited negative results. The SAR analysis indicated that the C-2, 4, 5 hydroxyl or methoxyl on the xanthone skeleton may influence the activities in resisting bacterial infection.

The antibacterial capacity of $\alpha$-mangostin (23), cowanin (24), fuscaxanthone A (32), 9-hydroxycalabaxanthone (77) (Figure 6) [183] against Staphylococcus aureus suggested that the increase of the unsaturated isoprenyl groups lowered the anti-bacterial ability. Boonnak et al. [40] reported that 1,3,7-trihydroxyxanthones with isoprenyl or geranyl side chain and 1,3,7-trioxygenated xanthone with geranyl side chain showed strong inhibitory activity on P. aeruginosa (a kind of Gram-negative bacteria). 
Allanxanthones A (52), D (78) and 1,3,6,7-tetrahydroxy-2-(3-methylbut-2-enyl)xanthone (79) (Figure 6) obtained from the stem bark of $A$. gabonensis [23] were studied for their antifungal ability against Candida krusei. Allanxanthone D (78) showed stronger antibiotic activity than the reference antibiotic ( $\mathrm{IC}_{50} \mu \mathrm{g} / \mathrm{mL}$ : $1.22,2.44,2.44,4.88$, for $52,78,79$, and the reference antibiotic nystatin, respectively), which indicated that the oxygen substitution at C-6, 7 and the prenyl substitution at C-4 may be the active units.

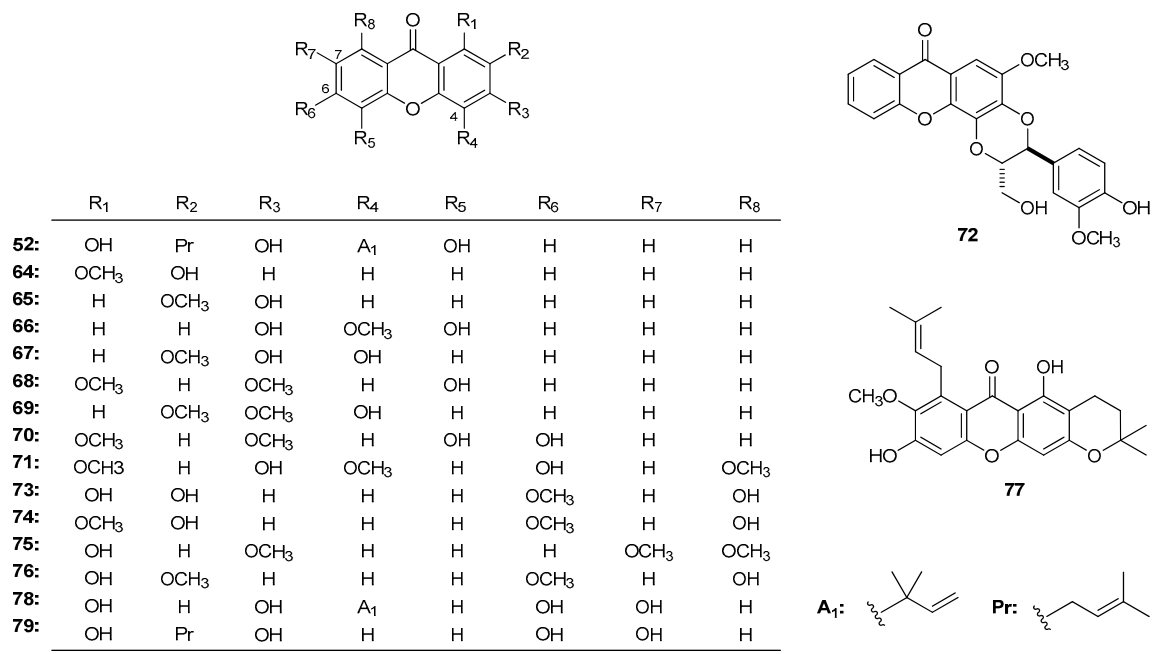

Figure 6. The structures of compounds 52, 64-79.

\section{4. $\alpha$-Glucosidase Inhibitory Activity}

Plants resources with $\alpha$-glucosidase inhibitory activity include the Cudrania [155], Garcinia [184], and Swertia [130] genera. Swertianolin (80), 1-O-[ $\beta$-D-xylopyranosyl- $(1 \rightarrow 6)-\beta-D-$ glucopyranosyl]-8-hydroxy-3,7-dimethoxyxanthone (81), kouitchenside D (82), mangiferin (83), kouitchensides F (84), B (85), E (86), kouitchenside I (87) (Figure 7) isolated from S. kouitchensis [130] showed inhibitory effects on $\alpha$-glucosidase with $\mathrm{IC}_{50}$ values of $126 \pm 23,451 \pm 41,360 \pm 39,296 \pm 52$, $184 \pm 23,383 \pm 18$ and $371 \pm 22 \mu \mathrm{M}$, inactive, respectively. The results revealed that the substitution with a primeverosyl residue led to increased inhibitory effects than other diglycoside units $(\mathbf{8 1}, \mathbf{8 2}, \mathbf{8 4}$, 85 , and $86>87)$, and oxygen substitution at C-1 or C-8 $(80,83$ and 84$)$, while a diglycoside residue located at C-7 (87) reduced the inhibitory activity.

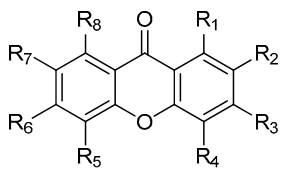

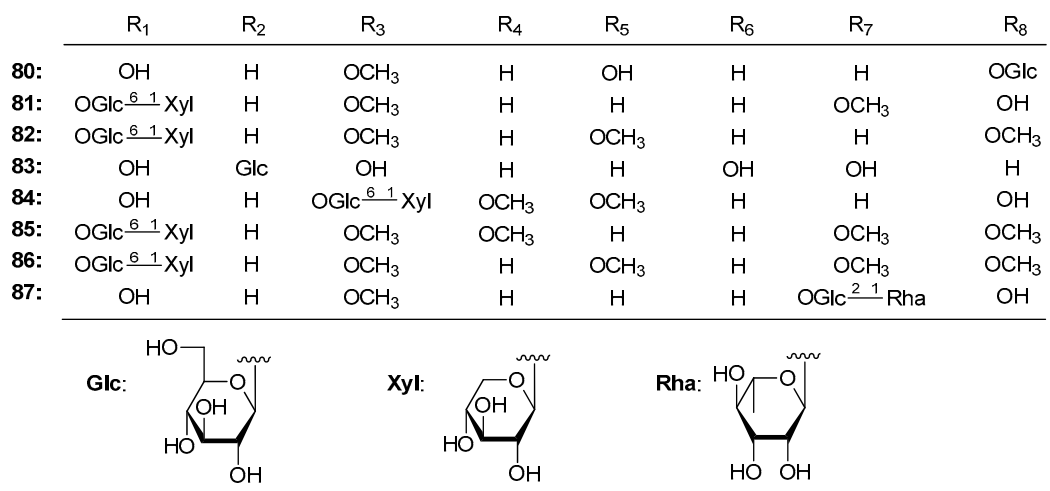

Figure 7. The structures of compounds 80-87. 


\subsection{Anti-Virus Activity}

Xanthones obtained from Comastoma [108], Garcinia [178], and Swertia [128] genera exhibited inhibitory effects on tobacco mosaic virus (TMV). The anti-TMV half-leaf tests on paucinervins $\mathrm{E}$ (88), F (89), G (90), cudraxanthone G (91), ananixanthone (92), merguenone (93), nigrolineaxanthone $\mathrm{K}$ (94), 5-O-methylxanthone V1 (95) (Figure 8) ( $\mathrm{IC}_{50}: 21.4 \pm 2.3,42.8 \pm 3.0,53.6 \pm 2.2,52.8 \pm 3.0$, $68.9 \pm 2.3,82.4 \pm 2.6 \mu \mathrm{M}$, for $\mathbf{8 8}, \mathbf{8 9}, \mathbf{9 0}, \mathbf{9 1}, \mathbf{9 2}, \mathbf{9 5}$, respectively) [107] suggested that the hydroxyl groups might be one of the active units $(\mathbf{8 8}>\mathbf{8 9}$ and $\mathbf{9 0})$. The introduction of the pyran ring $(\mathbf{9 2}, \mathbf{9 3}, \mathbf{9 4}$, and 95) or the interaction through hydrogen bonding with an isoprenyl group (91) would lower the inhibitory activities.<smiles>[R6]c1cc([R6])c2c(=O)c3c(O)c([R])c([R])c([R])c3oc2c1[R]</smiles>

\begin{tabular}{lcccccc} 
& $\mathrm{R}_{1}$ & $\mathrm{R}_{2}$ & $\mathrm{R}_{3}$ & $\mathrm{R}_{4}$ & $\mathrm{R}_{5}$ & $\mathrm{R}_{6}$ \\
\cline { 2 - 7 } 88: & $\mathrm{H}$ & $\mathrm{A}_{3}$ & $\mathrm{H}$ & $\mathrm{H}$ & $\mathrm{OH}$ & $\mathrm{COOCH}_{3}$ \\
89: & $\mathrm{H}$ & $\mathrm{A}_{3}$ & $\mathrm{H}$ & $\mathrm{H}$ & $\mathrm{OCH}_{3}$ & $\mathrm{COOCH}_{3}$ \\
90: & $\mathrm{H}$ & $\mathrm{A}_{3}$ & $\mathrm{H}$ & $\mathrm{OCH}_{3}$ & $\mathrm{H}$ & $\mathrm{COOCH}_{3}$ \\
91: & $\mathrm{Pr}$ & $\mathrm{OCH}_{3}$ & $\mathrm{Pr}$ & $\mathrm{OH}$ & $\mathrm{H}$ & $\mathrm{H}$
\end{tabular}

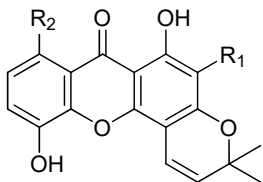

92: \begin{tabular}{ll}
$\mathrm{R}_{1}$ & $\mathrm{R}_{2}$ \\
\hline $\mathrm{Pr}$ & $\mathrm{H}$ \\
93: & $\mathrm{Pr}$ \\
\hline
\end{tabular}<smiles>CC(CO)C(O)CO</smiles>

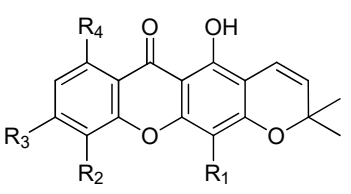

94: \begin{tabular}{llll}
$\mathrm{R}_{1}$ & $\mathrm{R}_{2}$ & $\mathrm{R}_{3}$ & $\mathrm{R}_{4}$ \\
\hline $\mathrm{H}$ & $\mathrm{OH}$ & $\mathrm{H}$ & $\mathrm{Pr}$
\end{tabular}

95: $\mathrm{Pr} \quad \mathrm{OCH}_{3} \quad \mathrm{OH} \quad \mathrm{H}$

Figure 8. The structures of compounds 88-95.

\subsection{Anti-Inflammatory Activity}

During the past ten years, plants belonging to the Artocarpus [154], Calophyllum [185], Cratoxylum [41], and Garcinia [46] genera have been reported to display anti-inflammatory activity. Pyranocycloartobiloxanthone A (96, Figure 9), a novel xanthone isolated from A. obtusus [154] presented not only anti-inflammatory and anti-oxidant activities, but also anti-apoptotic and anti-bacterial effects against Helicobacter pylori. The inhibitory effects of 1,7-dihydroxy-8-methoxyxanthone (97), cochinchinone A (98), formoxanthone A (99), macruraxanthone (4), cochinxanthone E (100), pruniflorone L (101), dulcisxanthone F (102), 5,9-dihydroxy-8-methoxy-2,2-dimethyl-7-(3-methyl-but-2-enyl)-2H,6H-pyrano-[3,2b]-xanthone (103), pruniflorone K (104), and garcinone B (105) (Figure 9) obtained from C. formosum ssp. pruniflorum showed inhibitory effects on NO production by murine macrophage-like RAW264.7 cells [41]. Among them, compounds $99,102,103$ displayed good suppressive ability on NO production with $\mathrm{IC}_{50}$ values of $8.0,3.9$, and $4.3 \mu \mathrm{M}$, respectively, while $\mathbf{9 8}, \mathbf{1 0 0}$ and $\mathbf{1 0 5}$ showed moderate activity with $\mathrm{IC}_{50}$ values of 12.6, 12.8 and $11.8 \mu \mathrm{M}$, respectively. The investigation indicated that tetraoxygenated xanthone skeleton exhibited inhibition of NO production greater than trioxygenated xanthone skeleton, while the methoxyl group at C-3 or C-7 on the tetraoxygenated isoprenylated-xanthone skeleton was an essential group.

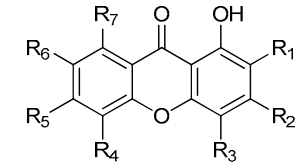

\begin{tabular}{cccccccc} 
& $\mathrm{R}_{1}$ & $\mathrm{R}_{2}$ & $\mathrm{R}_{3}$ & $\mathrm{R}_{4}$ & $\mathrm{R}_{5}$ & $\mathrm{R}_{6}$ & $\mathrm{R}_{7}$ \\
\cline { 2 - 7 } 97: & $\mathrm{H}$ & $\mathrm{H}$ & $\mathrm{H}$ & $\mathrm{H}$ & $\mathrm{H}$ & $\mathrm{OH}$ & $\mathrm{OCH}_{3}$ \\
98: & $\mathrm{Pr}$ & $\mathrm{OH}$ & $(E)-\mathrm{Ge}$ & $\mathrm{H}$ & $\mathrm{H}$ & $\mathrm{OH}$ & $\mathrm{H}$ \\
99: & $(E)-\mathrm{Ge}$ & $\mathrm{OH}$ & $\mathrm{Pr}$ & $\mathrm{OH}$ & $\mathrm{H}$ & $\mathrm{H}$ & $\mathrm{H}$ \\
100: & $\mathrm{Pr}$ & $\mathrm{OH}$ & $\mathrm{H}$ & $\mathrm{H}$ & $\mathrm{H}$ & $\mathrm{OH}$ & $(E)-\mathrm{Ge}$ \\
101: & $\mathrm{Pr}$ & $\mathrm{OCH}_{3}$ & $(E)-\mathrm{Ge}$ & $\mathrm{H}$ & $\mathrm{H}$ & $\mathrm{OH}$ & $\mathrm{H}$ \\
102: & $\mathrm{Pr}$ & $\mathrm{OCH}_{3}$ & $\mathrm{H}$ & $\mathrm{H}$ & $\mathrm{OH}$ & $\mathrm{OH}$ & $\mathrm{Pr}$ \\
\hline
\end{tabular}
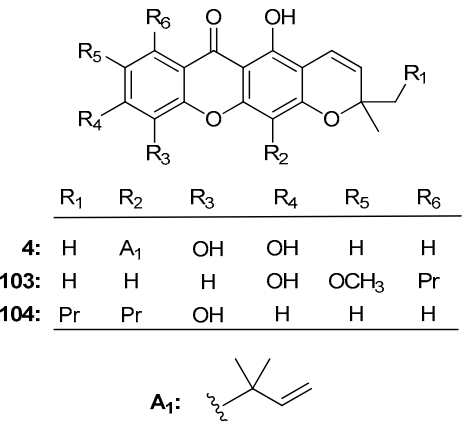<smiles></smiles>

96<smiles>CC(C)=CCc1c(O)cc2oc3cc(O)c4c(c3c(=O)c2c1O)CCC(C)(C)O4</smiles>

Figure 9. The structures of compounds 4, 96-105. 


\section{Conclusions}

As a class of secondary metabolites obtained from a number of herbal medicines, xanthones are playing more and more important roles in new drug research and development. The pharmacokinetics and toxicity (PK/tox) properties of xanthones, as part of the most crucial preclinical studies, have proved that xanthones are promising drug candidates owing to their high efficacy and low toxicity $[186,187]$.

In this paper, we have summarized the plant sources, bioactivity and the SARs of xanthones from literature published over the last few decades. As a result, 168 species of herbal medicine plants belonging to 58 genera, and 24 families were found to be enriched in xanthones. Among them, the Calophyllum, Cratoxylum, Cudrania, Garcinia, Gentiana, Hypericum and Swertia genera are the plant resource with the most development prospect. Xanthones display multiple bioactivities, which may be useful for new drug development for cancer, inflammation, bacterial, fungal and viral infection, diabetes, and so on.

Acknowledgments: Part of this research was supported by the Program of the National Natural Science Foundation of China (No. 81673688, 81673703), Natural Science Foundation of Tianjin City (No. 15JCYBJC54900), and Changjiang Scholars and Innovative Research Team in University (PCSIRT IRT_14R41).

Author Contributions: Yi Zhang and Tao Wang designed the manuscript; Jingya Ruan wrote the manuscript; Chang Zheng, Yanxia Liu, and Lu Qu retrieved the literature; Haiyang Yu and Lifeng Han perfected the language. All authors discussed, edited and approved the final version.

Conflicts of Interest: The authors declare no conflict of interest.

\section{References}

1. Vieira, L.M.; Kijjoa, A. Naturally-occurring xanthones: Recent developments. Curr. Med. Chem. 2005, 12, 2413-2446. [CrossRef] [PubMed]

2. Peres, V.; Nagem, T.J.; de Oliveira, F.F. Tetraoxygenated naturally occurring xanthones. Phytochemistry 2000, 55, 683-710. [CrossRef]

3. Han, Q.B.; Xu, H.X. Caged Garcinia xanthones: Development since 1937. Curr. Med. Chem. 2009, 16, 3775-3796. [CrossRef] [PubMed]

4. Gutierrez-Orozco, F.; Failla, M.L. Biological activities and bioavailability of mangosteen xanthones: A critical review of the current evidence. Nutrients 2013, 5, 3163-3183. [CrossRef] [PubMed]

5. Wang, X.; Chen, W. Gambogic Acid is a Novel Anti-cancer Agent that Inhibits Cell Proliferation, Angiogenesis and Metastasis. Anticancer Agents. Med. Chem. 2012, 12, 994-1000. [CrossRef] [PubMed]

6. Wang, J.; Liu, W.; Zhao, Q.; Qi, Q.; Lu, N.; Yang, Y.; Nei, F.F.; Rong, J.J.; You, Q.D.; Guo, Q.L. Synergistic effect of 5-fluorouracil with gambogic acid on BGC-823 human gastric carcinoma. Toxicology 2009, 256, 135-140. [CrossRef] [PubMed]

7. Yang, Y.; Yang, L.; You, Q.D.; Nie, F.F.; Gu, H.Y.; Zhao, L.; Wang, X.T.; Guo, Q.L. Differential apoptotic induction of gambogic acid, a novel anticancer natural product, on hepatoma cells and normal hepatocytes. Cancer Lett. 2007, 256, 259-266. [CrossRef] [PubMed]

8. Wang, T.; Wei, J.; Qian, X.; Ding, Y.; Yu, L.; Liu, B. Gambogic acid, a potent inhibitor of survivin, reverses docetaxel resistance in gastric cancer cells. Cancer Lett. 2008, 262, 214-222. [CrossRef] [PubMed]

9. Zhou, Z.T.; Wang, J.W. Phase I human tolerability trial of gambogic acid. Chin. J. New Drugs 2007, 1, 79-83.

10. Tang, Y.P.; Li, P.G.; Kondo, M.; Ji, H.P.; Kou, Y.; Ou, B. Effect of a mangosteen dietary supplement on human immune function: A randomized, double-blind, placebo-controlled trial. J. Med. Food 2009, 12, 755-763. [CrossRef] [PubMed]

11. Dua, V.K.; Verma, G.; Dash, A.P. In vitro antiprotozoal activity of some xanthones isolated from the roots of Andrographis paniculata. Phytother. Res. 2009, 23, 126-128. [CrossRef] [PubMed]

12. Ramirez, J.E.; Zambrano, R.; Sepúlveda, B.; Simirgiotis, M.J. Antioxidant properties and hyphenated HPLC-PDA-MS profiling of chilean pica mango fruits (Mangifera indica L. Cv. piqueño). Molecules 2013, 19, 438-458. [CrossRef] [PubMed]

13. Singh, O.; Ali, M.; Akhtar, N. New antifungal xanthones from the seeds of Rhus coriaria L. Z. Naturforsch. C 2011, 66, 17-23. [CrossRef] [PubMed] 
14. Sabphon, C.; Sermboonpaisarn, T.; Sawasdee, P. Cholinesterase inhibitory activities of xanthones from Anaxagorea luzonensis A. Gray. J. Med. Plant Res. 2012, 6, 3781-3785.

15. Emmanoel, V.C.; Assis, M.F.; Lúcia, B.M.; Braga, R.M. Chemical constituents isolated from the bark of Guatteria blepharophylla (Annonaceae) and their antiproliferative and antimicrobial activities. J. Braz. Chem. Soc. 2011, 22, 1111-1117.

16. Waller, C.P.; Thumser, A.E.; Langat, M.K.; Crouch, N.R.; Mulholland, D.A. COX-2 inhibitory activity of homoisoflavanones and xanthones from the bulbs of the Southern African Ledebouria socialis and Ledebouria ovatifolia (Hyacinthaceae: Hyacinthoideae). Phytochemistry 2013, 95, 284-290. [CrossRef] [PubMed]

17. Guo, J.; Xu, C.H.; Xue, R.; Jiang, W.X.; Wu, B.; Huang, C.G. Cytotoxic activities of chemical constituents from rhizomes of Anemarrhena asphodeloides and their analogues. Arch. Pharm. Res. 2015, 38, 598-603. [CrossRef] [PubMed]

18. Mulholland, D.A.; Koorbanally, C.; Crouch, N.R.; Sandor, P. Xanthones from Drimiopsis maculate. J. Nat. Prod. 2004, 67, 1726-1728. [CrossRef] [PubMed]

19. Cottiglia, F.; Casu, L.; Bonsignore, L.; Casu, M.; Floris, C.; Sosa, S.; Altinier, G.; Loggia, R.D. Topical anti-inflammatory activity of flavonoids and a new xanthone from Santolina insularis. Z. Naturforsch. C 2005, 60, 63-66. [CrossRef] [PubMed]

20. Pauletti, P.M.; Castro-Gamboa, I.; Silva, D.; Helena, S. New antioxidant C-glucosylxanthones from the stems of Arrabidaea samydoides. J. Nat. Prod. 2003, 66, 1384-1387. [CrossRef] [PubMed]

21. Sati, S.C.; Sati, M.D.; Sharma, A. Isolation and characterization of flavone di-glucoside and acetoxyxanthone from the flowers of Bombex ceiba. J. Appl. Nat. Sci. 2011, 3, 128-130.

22. Nkengfack, A.E.; Azebaze, G.A.; Vardamides, J.C.; Fomum, Z.T.; Heerden, F.R. A prenylated xanthone from Allanblackia floribunda. Phytochemistry 2002, 60, 381-384. [CrossRef]

23. Azebaze, A.G.B.; Ouahouo, B.M.W.; Vardamides, J.C.; Valentin, A.; Kuete, V.; Acebey, L.; Beng, V.P.; Nkengfack, A.E.; Meyer, M. Antimicrobial and antileishmanial xanthones from the stem bark of Allanblackia gabonensis. Chem. Nat. Compd. 2008, 44, 582-587. [CrossRef]

24. Azebaze, A.G.; Menasria, F.; Noumi, L.G.; Nguemfo, E.L. Xanthones from the seeds of Allanblackia monticola and their apoptotic and antiproliferative activities. Planta Med. 2009, 75, 243-248. [CrossRef] [PubMed]

25. Carvalho, M.P.; Lima, M.M.C.; Santos, M.G.; Rocha, L.M.; Kuster, R.M. Anthraquinones and xanthone from Bonnetia stricta and their chemosystematic significance. Biochem. Syst. Ecol. 2013, 48, 73-75. [CrossRef]

26. Blanco-Ayala, T.; Lugo-Huitron, R.; Serrano-Lopez, E.M.; Reyes-Chilpa, R.; Rangel-Lopez, E.; Pineda, B.; Medina-Campos, O.N.; Sanchez-Chapul, L.; Pinzon, E.; Cristina, T.S. Antioxidant properties of xanthones from Calophyllum brasiliense: Prevention of oxidative damage induced by $\mathrm{FeSO}_{4}$. BMC Complement. Altern. Med. 2013, 13, 262. [CrossRef] [PubMed]

27. Oger, J.M.; Morel, C.; Helesbeux, J.J.; Litaudon, M.; Seraphin, D.; Dartiguelongue, C.; Larcher, G.; Richomme, P.; Duval, O. First 2-hydroxy-3-methylbut-3-enyl substituted xanthones isolated from plants: Structure elucidation, synthesis and antifungal activity. Nat. Prod. Res. 2003, 17, 195-199. [CrossRef] [PubMed]

28. Ajithabai, M.D.; Rameshkumar, B.; Jayakumar, G.; Varma, L.; Nair, M.S.; Ajaikumar Nair, G.P. Decipic acid and 12-acetyl apetalic acid from Calophyllum decipiens Wight. Indian J. Chem. B 2012, 51B, 393-397.

29. Nasir, N.M.; Rahmani, M.; Shaari, K.; Kassim, N.K.; Go, R.; Stanslas, J.; Jeyaraj, E.J. Xanthones from Calophyllum gracilipes and their cytotoxic activity. Sains Malays. 2013, 42, 1261-1266.

30. Iinuma, M.; Tosa, H.; Tanaka, T.; Yonemori, S. Two xanthones from roots of Calophyllum inophyllum. Phytochemistry 1995, 38, 725-728. [CrossRef]

31. Ming, M.; Zhang, X.; Chen, H.F.; Zhu, L.J.; Zeng, D.Q.; Yang, J.; Wu, G.X.; Wu, Y.Z.; Yao, X.S. RXR $\alpha$ transcriptional inhibitors from the stems of Calophyllum membranaceum. Fitoterapia 2016, 108, 66-72. [CrossRef] [PubMed]

32. Ito, C.; Miyamoto, Y.; Rao, K.S.; Furukawa, H. A novel dibenzofuran and two new xanthones from Calophyllum panciflorum. Chem. Pharm. Bull. 1996, 44, 441-443. [CrossRef]

33. Alarcon, A.B.; Cuesta-Rubio, O.; Cardenas Perez, J.; Piccinelli, A.L.; Rastrelli, L. Constituents of the Cuban endemic species Calophyllum pinetorum. J. Nat. Prod. 2008, 71, 1283-1286. [CrossRef] [PubMed]

34. Mah, S.H.; Ee, G.C.; The, S.S.; Rahmani, M.; Lim, Y.M.; Go, R. Phylattrin, a new cytotoxic xanthone from Calophyllum soulattri. Molecules 2012, 17, 8303-8311. [CrossRef] [PubMed] 
35. Kawamura, F.; Muhamud, A.; Hashim, R.; Sulaiman, O.; Ohara, S. Two antifungal xanthones from the heartwood of Calophyllum symingtonianum. Jpn. Agric. Res. 2012, 46, 181-185. [CrossRef]

36. Nguyen, L.T.; Nguyen, D.M.; Nguyen, L.H.D. A new xanthone from the bark of Calophyllum thorelii. Nat. Prod. Res. 2013, 27, 563-567. [CrossRef] [PubMed]

37. Dharmaratne, H.R.W.; Wanigasekera, W.M.A.P. Xanthones from root bark of Calophyllum thwaitesii. Phytochemistry 1996, 42, 249-250. [CrossRef]

38. Molinar-Toribio, E.; Gonzalez, J.; Ortega-Barria, E.; Capson, T.L.; Coley, P.D.; Kursar, T.A.; McPhail, K.; Cubilla-Rios, L. Antiprotozoal activity against Plasmodium falciparum and Trypanosoma cruzi of xanthones isolated from Chrysochlamys tenuis. Pharm. Biol. 2006, 44, 550-553. [CrossRef]

39. Silva, E.M.; Araujo Renata, M.; Freire-Filha, L.G.; Silveira, E.R.; Lopes, N.P.; Elias, P.J.; Braz-Filho, R.; Espindola, L.S. Clusiaxanthone and tocotrienol series from Clusia pernambucensis and their antileishmanial activity. J. Braz. Chem. Soc. 2013, 24, 1314-1321.

40. Wilawan, M.; Rattanaburi, S.; Phongpaichit, S.; Kanjana-Opas, A. Antibacterial and cytotoxic xanthones from Cratoxylum cochinchinense. Phytochem. Lett. 2008, 1, 211-214.

41. Boonnak, N.; Chantrapromma, S.; Tewtrakul, S.; Sudsai, T. Inhibition of nitric oxide production in lipopolysaccharide-activated RAW264.7 macrophages by isolated xanthones from the roots of Cratoxylum formosum ssp. pruniflorum. Arch. Pharm. Res. 2014, 37, 1329-1335. [CrossRef] [PubMed]

42. Kamdem, W.; Mulholland, D.; Wansi, J.D.; Mbaze, L.M.; Powo, R. Afzeliixanthones A and B, 2 new prenylated xanthones from Garcinia afzelii ENGL. (Guttiferae). Chem. Pharm. Bull. 2006, 54, 448-451. [CrossRef]

43. Niu, S.L.; Li, Z.L.; Ji, F.; Liu, G.Y.; Zhao, N.; Liu, X.Q.; Jing, Y.K.; Hua, H.M. Xanthones from the stem bark of Garcinia bracteata with growth inhibitory effects against HL-60 cells. Phytochemistry 2012, 77, 280-286. [CrossRef] [PubMed]

44. Milena, M.; Bassarello, C.; Bifulco, G.; Piacente, S. Polyisoprenylated benzophenone derivatives from the fruits of Garcinia cambogia and their absolute configuration by quantum chemical circular dichroism calculations. Tetrahedron 2010, 66, 139-145.

45. Jantan, I.; Saputri, F.C. Benzophenones and xanthones from Garcinia cantleyana var. cantleyana and their inhibitory activities on human low-density lipoprotein oxidation and platelet aggregation. Phytochemistry 2012, 80, 58-63. [PubMed]

46. Ritthiwigrom, T.; Laphookhieo, S.; Pyne, S.G. Chemical constituents and biological activities of Garcinia cowa Roxb. Maejo. Int. J. Sci. Technol. 2013, 7, 212-231.

47. Iinuma, M.; Tosa, H.; Tanaka, T.; Riswan, S. Three new xanthones from the bark of Garcinia dioica. Chem. Pharm. Bull. 1996, 44, 232-234. [CrossRef]

48. Deachathai, S.; Mahabusarakam, W.; Phongpaichit, S.; Taylor, W.C. Phenolic compounds from the fruit of Garcinia dulcis. Phytochemistry 2005, 66, 2368-2375. [CrossRef] [PubMed]

49. Mian, J.V.Y.; Lian, E.G.C.; Aspollah, S.M.; Hin, T.Y.Y.; Yen, K.H.; Yok, C.M.K. Benzophenone constituents from the roots of Garcinia eugenifolia. Res. J. Chem. Environ. 2012, 16, 36-39.

50. Ito, C.; Itoigawa, M.; Takakura, T.; Ruangrungsi, N.; Enjo, F.; Tokuda, H.; Nishino, H.; Furukawa, H. Chemical constituents of Garcinia fusca: Structure elucidation of eight new xanthones and their cancer chemopreventive activity. J. Nat. Prod. 2003, 66, 200-205. [CrossRef] [PubMed]

51. Mahamodo, S.; Riviere, C.; Neut, C.; Abedini, A.; Ranarivelo, H.; Duhal, N.; Roumy, V. Antimicrobial prenylated benzoylphloroglucinol derivatives and xanthones from the leaves of Garcinia goudotiana. Phytochemistry 2014, 102, 162-168. [CrossRef] [PubMed]

52. Alkadi, K.A.A.; Adam, A.; Taha, M.; Hasan, M.H.; Shah, S.A.A. Prenylated xanthone and rubraxanthone with antiplatelet aggregation activity in human whole blood isolated from Garcinia griffithii. Orient. J. Chem. 2013, 29, 1291-1295. [CrossRef]

53. Deng, Y.X.; Pan, S.L.; Zhao, S.Y.; Wu, M.Q.; Sun, Z.Q.; Chen, X.H.; Shao, Z.Y. Cytotoxic alkoxylated xanthones from the resin of Garcinia hanburyi. Fitoterapia 2012, 83, 1548-1552. [CrossRef] [PubMed]

54. Klaiklay, S.; Sukpondma, Y.; Rukachaisirikul, V.; Phongpaichit, S. Friedolanostanes and xanthones from the twigs of Garcinia hombroniana. Phytochemistry 2013, 85, 161-166. [CrossRef] [PubMed]

55. Sun, Y.; Li, D.; Jia, C.C.; Xue, C.M.; Bai, J.; Li, Z.L.; Hua, H.M. Three new xanthones from the leaves of Garcinia lancilimba. J. Nat. Med. 2016, 70, 173-178. [CrossRef] [PubMed] 
56. Ren, Y.; Lantvit, D.D.; Carcache, E.J.; Kardono, L.B.S.; Riswan, S. Proteasome-inhibitory and cytotoxic constituents of Garcinia lateriflora: Absolute configuration of caged xanthones. Tetrahedron 2010, 66, 5311-5320. [CrossRef] [PubMed]

57. Chen, J.J.; Chen, I.S.; Duh, C.Y. Cytotoxic xanthones and biphenyls from the root of Garcinia linii. Planta Med. 2004, 70, 1195-1200. [CrossRef] [PubMed]

58. Chae, H.S.; Kim, E.Y.; Han, L.; Kim, N.R.; Lam, B.; Paik, J.H.; Yoon, K.D. Xanthones with pancreatic lipase inhibitory activity from the pericarps of Garcinia mangostana L. (Guttiferae). Eur. J. Lipid Sci. Technol. 2016, 118, 1416-1421. [CrossRef]

59. Nguyen, L.H.D.; Vo, H.T.; Pham, H.D.; Connolly, J.D.; Harrison, L.J. Xanthones from the bark of Garcinia merguensis. Phytochemistry 2003, 63, 467-470. [CrossRef]

60. Chiang, Y.M.; Kuo, Y.H.; Oota, S.; Fukuyama, Y. Xanthones and benzophenones from the stems of Garcinia multiflora. J. Nat. Prod. 2003, 66, 1070-1073. [CrossRef] [PubMed]

61. Rukachaisirikul, V.; Ritthiwigrom, T.; Pinsa, A.; Sawangchote, P.; Taylor, W.C. Xanthones from the stem bark of Garcinia nigrolineata. Phytochemistry 2003, 64, 1149-1156. [CrossRef]

62. Ee, G.C.L.; Foo, C.H.; Jong, V.Y.M.; Ismail, N.H.; Sukari, M.A.; Ya, Y.H.T.; Awang, K. A new xanthone from Garcinia nitida. Nat. Prod. Res. 2012, 26, 830-835. [CrossRef] [PubMed]

63. Fouotsa, H.; Tatsimo, S.J.N.; Neumann, B.; Michalek, C.; Mbazoa, C.D.; Nkengfack, A.E.; Sewald, N.; Lannang, A.M. A new xanthone derivative from twigs of Garcinia nobilis. Nat. Prod. Res. 2014, 28, 1030-1036. [CrossRef] [PubMed]

64. Tang, Z.; Xia, Z.X.; Qiao, S.P.; Jiang, C.; Shen, G.R.; Cai, M.X.; Tang, X.Y. Four new cytotoxic xanthones from Garcinia nujiangensis. Fitoterapia 2015, 102, 109-114. [CrossRef] [PubMed]

65. Shan, W.G.; Lin, T.D.; Yu, H.N.; Chen, Y.; Zhan, Z.J. Polyprenylated xanthones and benzophenones from the bark of Garcinia oblongifolia. Helv. Chim. Acta 2012, 95, 1442-1448. [CrossRef]

66. Tang, Y.X.; Fu, W.W.; Wu, R.; Tan, H.S.; Shen, Z.; Xu, H.X. Bioassay-guided isolation of prenylated xanthone derivatives from the leaves of Garcinia oligantha. J. Nat. Prod. 2016, 79, 1752-1761. [CrossRef] [PubMed]

67. Ha, L.D.; Hansen, P.E.; Vang, O.; Duus, F.; Pham, H.D.; Nguyen, L.H.D. Cytotoxic geranylated xanthones and O-alkylated derivatives of $\alpha$-mangostin. Chem. Pharm. Bull. 2009, 57, 830-834. [CrossRef] [PubMed]

68. Kardono, L.B.S.; Hanafi, M.; Sherley, G.; Kosela, S.; Harrison, L.J. Bioactive constituents of Garcinia porrecta and G. parvifolia grown in Indonesia. Pak. J. Biol. Sci. 2006, 9, 483-486.

69. Wu, Y.P.; Zhao, W.; Xia, Z.Y.; Kong, G.H.; Lu, X.P.; Hu, Q.F.; Gao, X.M. Three novel xanthones from Garcinia paucinervis and their anti-TMV activity. Molecules 2013, 18, 9663-9669. [CrossRef] [PubMed]

70. Vo, H.T.; Nguyen, N.T.T.; Maas, G.; Werz, U.R.; Pham, H.D.; Nguyen, L.H.D. Xanthones from the bark of Garcinia pedunculata. Phytochem. Lett. 2012, 5, 766-769. [CrossRef]

71. Jabit, M.L.; Khalid, R.; Abas, F.; Shaari, K.; Hui, L.S.; Stanslas, J.; Lajis, N.H. Cytotoxic xanthones from Garcinia penangiana Pierre. Z. Naturforsch. C 2007, 62, 786-792. [CrossRef] [PubMed]

72. Lannang, A.M.; Komguem, J.; Ngninzeko, F.N.; Tangmouo, J.G.; Lontsi, D.; Ajaz, A.; Choudhary, M.I.; Ranjit, R.; Devkota, K.P.; Sondengam, B.L. Bangangxanthone A and B, two xanthones from the stem bark of Garcinia polyantha Oliv. Phytochemistry 2005, 66, 2351-2355. [CrossRef] [PubMed]

73. Tantapakul, C.; Phakhodee, W.; Ritthiwigrom, T.; Cheenpracha, S.; Prawat, U.; Deachathai, S.; Laphookhieo, S. Rearranged benzophenones and prenylated xanthones from Garcinia propinqua Twigs. J. Nat. Prod. 2012, 75, 1660-1664. [CrossRef] [PubMed]

74. Elya, B.; He, H.P.; Kosela, S.; Hanafi, M.; Hao, X.J. A new cytotoxic xanthone from Garcinia rigida. Fitoterapia 2008, 79, 182-184. [CrossRef] [PubMed]

75. Sukandar, E.R.; Siripong, P.; Khumkratok, S.; Tip-pyang, S. New depsidones and xanthone from the roots of Garcinia schomburgkiana. Fitoterapia 2016, 111, 73-77. [CrossRef] [PubMed]

76. Sukpondma, Y.; Rukachaisirikul, V.; Phongpaichit, S. Xanthone and sesquiterpene derivatives from the fruits of Garcinia scortechinii. J. Nat. Prod. 2005, 68, 1010-1017. [CrossRef] [PubMed]

77. Fouotsa, H.; Lannang, A.M.; Dzoyem, J.P.; Tatsimo, S.J.N.; Neumann, B.; Mbazoa, C.D. Antibacterial and antioxidant xanthones and benzophenone from Garcinia smeathmannii. Planta Med. 2015, 81, 594-599. [CrossRef] [PubMed]

78. Ngoupayo, J.; Tabopda, T.K.; Ali, M.S. Antimicrobial and immunomodulatory properties of prenylated xanthones from twigs of Garcinia staudtii. Bioorg. Med. Chem. 2009, 17, 5688-5695. [CrossRef] [PubMed] 
79. Abe, F.; Nagafuji, S.; Okabe, H.; Higo, H.; Akahane, H. Trypanocidal constituents in plants 2. Xanthones from the stem bark of Garcinia subelliptica. Biol. Pharm. Bull. 2003, 26, 1730-1733. [CrossRef] [PubMed]

80. Duangsrisai, S.; Choowongkomon, K.; Bessa, L.J.; Costa, P.M.; Amat, N.; Kijjoa, A. Antibacterial and EGFR-tyrosine kinase inhibitory activities of polyhydroxylated xanthones from Garcinia succifolia. Molecules 2014, 19, 19923-19934. [CrossRef] [PubMed]

81. Guo, Y.E.; Wang, L.L.; Li, Z.L.; Niu, S.L.; Liu, X.Q.; Hua, H.M.; Chen, H.; Chu, J.; Zhang, T.C. Triterpenes and xanthones from the stem bark of Garcinia tetralata. J. Asian Nat. Prod. Res. 2011, 13, 440-443. [CrossRef] [PubMed]

82. Hay, A.E.; Aumond, M.C.; Mallet, S.; Dumontet, V.; Litaudon, M.; Rondeau, D.; Richomme, P. Antioxidant Xanthones from Garcinia vieillardii. J. Nat. Prod. 2004, 67, 707-709. [CrossRef] [PubMed]

83. Merza, J.; Aumond, M.C.; Rondeau, D.; Dumontet, V.; Le Ray, A.M. Prenylated xanthones and tocotrienols from Garcinia virgate. Phytochemistry 2004, 65, 2915-2920. [CrossRef] [PubMed]

84. Zhong, F.F.; Chen, Y.; Wang, P.; Feng, H.J.; Yang, G.Z. Xanthones from the bark of Garcinia xanthochymus and their 1,1-diphenyl-2-picrylhydrazyl radical-scavenging activity. Chin. J. Chem. 2009, 27, 74-80. [CrossRef]

85. Han, Q.B.; Yang, N.Y.; Tian, H.L.; Qiao, C.F.; Song, J.Z.; Chang, D.C.; Chen, S.L.; Luo, K.Q.; Xu, H.X. Xanthones with growth inhibition against HeLa cells from Garcinia xipshuanbannaensis. Phytochemistry 2008, 69, 2187-2192. [CrossRef] [PubMed]

86. Biesdorf, C.; Cortez, D.A.G.; Audi, E.A. Assessment of anxiolytic and panicolytic effects of dichloromethane fraction from stems of Kielmeyera coriacea. Phytomedicine 2012, 19, 374-377. [CrossRef] [PubMed]

87. Coqueiro, A.; Choi, Y.H.; Verpoorte, R.; Gupta, K.B.S.S.; De Mieri, M.; Hamburger, M.; Young, M.C.M.; Stapleton, P.; Gibbons, S.; Bolzani, V.S. Antistaphylococcal prenylated acylphoroglucinol and xanthones from Kielmeyera variabilis. J. Nat. Prod. 2016, 79, 470-476. [CrossRef] [PubMed]

88. Laphookhieo, S.; Promnart, P.; Syers, J.K.; Kanjana-Opas, A.; Ponglimanont, C.; Karalai, C. Coumarins and xanthones from the seeds of Mammea siamensis. J. Braz. Chem. Soc. 2007, 18, 1077-1080. [CrossRef]

89. Ee, G.C.L.; The, S.S.; Rahmani, M.; Taufiq-Yap, Y.H.; Go, R.; Mah, S.H. A new furanoxanthone from the root bark of Mesua ferrea. Lett. Org. Chem. 2012, 9, 457-459.

90. Karunakaran, T.; Ee, G.C.L.; The, S.S.; Daud, S.; Mah, S.H.; Lim, C.K.; Jong, V.Y.M.; Awang, K. A new coumarin from stem bark of Mesua hexapetala. Nat. Prod. Res. 2016, 30, 1591-1597. [CrossRef] [PubMed]

91. Maurice, T.; Marlyse, O.W.B.; Robert, N.J.; Pierre, M.; Victor, K.; Sterner, O.; Michelle, M.; Ephrem, N.A. Antimicrobial prenylated xanthones and anthraquinones from barks and fruits of Psorospermum adamauense (Engl.). Nat. Prod. J. 2013, 3, 60-65. [CrossRef]

92. Abou-Shoer, M.; Suwanborirux, K.; Habib, A.A.M.; Chang, C.J.; Cassady, J.M. Xanthones and vismiones from Psorospermum febrifugum. Phytochemistry 1993, 34, 1413-1420. [CrossRef]

93. Leet, J.E.; Liu, X.H.; Drexler, D.M.; Cantone, J.L.; Huang, S.; Mamber, S.W.; Fairchild, C.R.; Hussain, R.; Newman, D.J.; Kingston, D.G.I. Cytotoxic xanthones from Psorospermum molluscum from the madagascar rain forest. J. Nat. Prod. 2008, 71, 460-463. [CrossRef] [PubMed]

94. Almanza, G.R.; Quispe, R.; Mollinedo, P.; Rodrigo, G.; Fukushima, O.; Villagomez, R.; Akesson, B.; Sterner, O. Antioxidant and antimutagenic polyisoprenylated benzophenones and xanthones from Rheedia acuminate. Nat. Prod. Commun. 2011, 6, 1269-1274. [PubMed]

95. Cottet, K.; Neudorffer, A.; Kritsanida, M.; Michel, S.; Lallemand, M.C.; Largeron, M. Polycyclic polyprenylated xanthones from Symphonia globulifera: Isolation and biomimetic electrosynthesis. J. Nat. Prod. 2015, 78, 2136-2140. [CrossRef] [PubMed]

96. Tala, M.F.; De Dien Tamokou, J.; Tchakam, P.D.; Tane, P.; Kuiate, J.R.; Wabo, H.K. Antioxidant xanthones, anthraquinones and semi-synthetic derivatives from Vismia rubescens and Vismia laurentii. Pharmacologyonline 2011, 3, 1410-1418.

97. Zhao, H.; Wang, X.; Li, W.; Koike, K.; Bai, H. A new minor homoisoflavonoid from Caesalpinia sappan. Nat. Prod. Res. 2014, 28, 102-105. [CrossRef] [PubMed]

98. Sob, S.V.T.; Wabo, H.K.; Tane, P.; Ngadjui, B.T.; Ma, D. A xanthone and a polyketide derivative from the leaves of Cassia obtusifolia (Leguminosae). Tetrahedron 2008, 64, 7999-8002. [CrossRef]

99. Kokotkiewicz, A.; Luczkiewicz, M.; Pawlowska, J.; Luczkiewicz, P.; Sowinski, P.; Witkowski, J.; Bryl, E.; Bucinski, A. Isolation of xanthone and benzophenone derivatives from Cyclopia genistoides (L.) Vent. (honeybush) and their pro-apoptotic activity on synoviocytes from patients with rheumatoid arthritis. Fitoterapia 2013, 90, 199-208. [CrossRef] [PubMed] 
100. Li, W.; Sun, Y.N.; Yan, X.T.; Yang, S.Y.; Choi, C.W.; Kim, Y.H. Phenolic compounds from Desmodium caudatum. Nat. Prod. Sci. 2013, 19, 215-220.

101. Rezanka, T.; Jachymova, J.; Dembitsky, V.M. Prenylated xanthone glucosides from Ural's lichen Umbilicaria proboscidea. Phytochemistry 2003, 62, 607-612. [CrossRef]

102. El-Shanawany, M.A.; Mohamed, G.A.; Nafady, A.M.; Ibrahim, S.R.M.; Radwan, M.M.; Ross, S.A. A new xanthone from the roots of Centaurium spicatum. Phytochem. Lett. 2011, 4, 126-128. [CrossRef]

103. Zhou, M.; Zhou, K.; Zhao, Y.L.; Xiang, N.J.; Zhang, T.D.; Wang, Y.D.; Dong, W.; Ji, B.K.; Li, L.M.; Lou, J. Three new prenylated xanthones from Comastoma pedunculatum and their anti-tobacco mosaic virus activity. Phytochem. Lett. 2015, 11, 245-248. [CrossRef]

104. Zhou, M.; Zhou, K.; Zhao, Y.L.; Xiang, N.J.; Zhang, T.D.; Wang, Y.D.; Zhang, C.M.; Wang, Y.D.; Dong, W.; Ji, B.K.; et al. New xanthones from Comastoma pulmonarium and their anti-tobacco mosaic virus activity. Heterocycles 2015, 91, 604-609.

105. Krstic-Milosevic, D.; Jankovic, T.; Vinterhalter, B.; Menkovic, N.; Aljancic, I.; Vinterhalter, D. Influence of carbohydrate source on xanthone content in root cultures of Gentiana dinarica Beck. Plant Growth Regul. 2013, 71, 147-155. [CrossRef]

106. Isakovic, A.; Jankovic, T.; Harhaji, L.; Kostic-Rajacic, S.; Nikolic, Z.; Vajs, V.; Trajkovic, V. Antiglioma action of xanthones from Gentiana kochiana: Mechanistic and structure-activity requirements. Bioorg. Med. Chem. 2008, 16, 5683-5694. [CrossRef] [PubMed]

107. Aberham, A.; Schwaiger, S.; Stuppner, H.; Ganzera, M. Quantitative analysis of iridoids, secoiridoids, xanthones and xanthone glycosides in Gentiana lutea L. roots by RP-HPLC and LC-MS. J. Pharm. Biomed. Anal. 2007, 45, 437-442. [CrossRef] [PubMed]

108. Tian, X.; Xu, X.Z.; Zhan, H.Y.; Wang, A.L. Two new O- and C-glycosylxanthones from Gentiana tizuensis Franch. Indian J. Chem. B 2003, 42B, 950-952.

109. Jankovic, T.; Krstic-Milosevic, D.; Aljancic, I.; Savikin, K.; Menkovic, N.; Radanovic, D.; Milosavljevic, S. Phytochemical re-investigation of Gentiana utriculosa. Nat. Prod. Res. 2009, 23, 466-469. [PubMed]

110. Petrovic, S.; Leskovac, A.; Joksic, G. Radioprotective properties of Gentiana dinarica polyphennols on human lymphocytes in vitro. Res. Artic. 2008, 95, 1035-1041.

111. Lv, L.J.; Li, M.H. Terpenoilds, flavonoids and xanthones from Gentianella acuta (Gentianaceae). Biochem. Syst. Ecol. 2009, 37, 497-500.

112. Urbain, A.; Marston, A.; Batsuren, D.; Purev, O.; Hostettmann, K. Preparative isolation of closely-related xanthones from Gentianella amarella ssp. acuta by high-speed countercurrent chromatography. Phytochem. Anal. 2008, 19, 514-519. [CrossRef] [PubMed]

113. Zhang, L.; Zou, D.Z.; Bai, S.; Li, Z.H.; Zhang, C.H.; Li, M.H. Chemical constituents from Gentianella turkestanorum (Gentianaceae). Biochem. Syst. Ecol. 2016, 65, 89-92. [CrossRef]

114. Cui, Z.H.; Li, Y.; Wang, Z.W.; Zhang, J.; Zhang, C.H.; Zhang, N.; Li, M.H. Chemical constituents from Gentianopsis barbata var. sinensis Ma (Gentianaceae). Biochem. Syst. Ecol. 2013, 47, 101-103. [CrossRef]

115. Yeung, M.F.; Lau, C.B.S.; Chan, R.C.Y.; Zong, Y.Y.; Che, C.T. Search for antimycobacterial constituents from a Tibetan medicinal plant, Gentianopsis paludosa. Phytother. Res. 2009, 23, 123-125. [CrossRef] [PubMed]

116. Rodriguez, S.; Wolfender, J.L.; Odontuya, G.; Purev, O.; Hostettmann, K. Xanthones, secoiridoids and flavonoids from Halenia corniculata. Phytochemistry 1995, 40, 1265-1272. [CrossRef]

117. Wang, Y.; Shi, J.G.; Wang, M.Z.; Che, C.T.; Yeung, J.H.K. Vasodilatory actions of xanthones isolated from a Tibetan herb, Halenia elliptica. Phytomedicine 2009, 16, 1144-1150. [PubMed]

118. Li, L.; Li, M.H.; Zhang, N.; Huang, L.Q. Chemical constituents from Lomatogonium carinthiacum (Gentianaceae). Biochem. Syst. Ecol. 2011, 39, 766-768. [CrossRef]

119. Terreaux, C.; Maillard, M.; Gupta, M.P.; Hostettmann, K. Xanthones from Schultesia lisianthoides. Phytochemistry 1995, 40, 1791-1795. [CrossRef]

120. Singh, P.P.; Ambika Chauhan, S.M.S. Activity-guided isolation of antioxidant xanthones from Swertia chirayita (Roxb.) H. Karsten (Gentianaceae). Nat. Prod. Res. 2012, 26, 1682-1686. [CrossRef] [PubMed]

121. Rahman, A.U.; Pervin, A.; Feroz, M.; Choudhary, M.I.; Qureshi, M.M.; Perveen, S.; Mir, I.; Khan, M.I. Phytochemical studies on Swertia cordata. J. Nat. Prod. 1994, 57, 134-137. [CrossRef]

122. Uvarani, C.; Arumugasamy, K.; Chandraprakash, K.; Sankaran, M.; Ata, A.; Mohan, P.S. A new DNA-intercalative cytotoxic allylic xanthone from Swertia corymbosa. Chem. Biodivers. 2015, 12, 358-370. [CrossRef] [PubMed] 
123. Khetwal, K.S.; Rajput, P.S.; Sajwan, K.; Pathak, S.; Adhikari, A. 1,5-Dihydroxy-3,8-dimethoxyxanthone from Swertia cuneata. Indian J. Chem. B 2003, 42B, 953-955. [CrossRef]

124. Jiang, W.; Zhu, D.L.; Wang, M.F.; Yang, Q.S.; Zuo, M.Y.; Zeng, L.; Li, G.P. Xanthones from the herb of Swertia elata and their anti-TMV activity. Nat. Prod. Res. 2015, 30, 1810-1815. [CrossRef] [PubMed]

125. Sun, Y.G.; Zhang, X.; Xue, X.Y.; Zhang, Y.; Xiao, H.B.; Liang, X.M. Rapid identification of polyphenol C-glycosides from Swertia franchetiana by HPLC-ESI-MS-MS. J. Chromatogr. Sci. 2009, 47, 190-196. [CrossRef] [PubMed]

126. Wan, L.S.; Min, Q.X.; Wang, Y.L.; Yue, Y.D.; Chen, J.C. Xanthone glycoside constituents of Swertia kouitchensis with $\alpha$-glucosidase inhibitory activity. J. Nat. Prod. 2013, 76, 1248-1253. [CrossRef] [PubMed]

127. Hajimehdipoor, H.; Dijoux-Franca, M.G.; Mariotte, A.M.; Amanzadeh, Y.; Sadat-Ebrahimi, S.E.; Ghazi-Khansari, M. Two new xanthone diglycosides from Swertia longifolia Boiss. Nat. Prod. Res. 2006, 20, 1251-1255. [CrossRef] [PubMed]

128. Uvarani, C.; Chandraprakash, K.; Sankaran, M.; Ata, A.; Mohan, P.S. Antioxidant and structure-activity relationships of five tetraoxygenated xanthones from Swertia minor (Griscb.) Knobl. Nat. Prod. Res. 2012, 26, 1265-1270. [CrossRef] [PubMed]

129. Luo, C.T.; Mao, S.S.; Liu, F.L.; Yang, M.X.; Chen, H.R.; Kurihara, H.; Li, Y.L. Antioxidant xanthones from Swertia mussotii, a high altitude plant. Fitoterapia 2013, 91, 140-147. [CrossRef] [PubMed]

130. Pant, N.; Misra, H.; Jain, D.C. A xanthone glycoside from aerial parts of Swertia paniculata. J. Saudi Chem. Soc. 2014, 18, 551-554. [CrossRef]

131. Li, J.C.; Feng, L.; Sun, B.H.; Ikeda, T.; Nohara, T. Hepatoprotective activity of the constituents in Swertia pseudochinensis. Biol. Pharm. Bull. 2005, 28, 534-537. [CrossRef] [PubMed]

132. Zheng, X.Y.; Yang, Y.F.; Li, W.; Zhao, X.; Sun, Y.; Sun, H.; Wang, Y.H.; Pu, X.P. Two xanthones from Swertia punicea with hepatoprotective activities in vitro and in vivo. J. Ethnopharmacol. 2014, 153, 854-863. [CrossRef] [PubMed]

133. Rana, V.S.; Rawat, M.S. A new xanthone glycoside and antioxidant constituents from the rhizomes of Swertia speciosa. Chem. Biodivers. 2005, 2, 1310-1315. [CrossRef] [PubMed]

134. Silva, L.F.; Luiz, F.; Lima, E.S.; Vasconcello, M.C. In vitro and in vivo antimalarial activity and cytotoxicity of extracts, fractions and a substance isolated from the Amazonian plant Tachia grandiflora (Gentianaceae). Memórias Instituto Oswaldo Cruz 2013, 108, 501-507. [CrossRef] [PubMed]

135. Periyar, S.S.; Magesh, V.; Raja, V.; Mahendran, T.S.; Muthumary, J.; Kalaichelvan, P.T.; Murugesan, K. Antimutagenicity of mangiferin purified from Salacia chinensis Linn. Int. Multidiscip. Res. J. 2011, 1, 1-5.

136. Duarte, L.P.; Figueiredo, R.C.; Faria De Sousa, G.; Soares, D.B.S.; Rodrigues, S.B.V.; Silva, F.C.; Silva, G.D.F.; Filho, S.A.V. Chemical constituents of Salacia elliptica (Celastraceae). Quim. Nova 2010, 33, 900-903. [CrossRef]

137. Nishida, Y.; Eto, M.; Miyashita, H.; Ikeda, T.; Yamaguchi, K.; Yoshimitsu, H.; Nohara, T.; Ono, M. A new homostilbene and two new homoisoflavones from the bulbs of Scilla scilloides. Chem. Pharm. Bull. 2008, 56, 1022-1025. [CrossRef] [PubMed]

138. Hashida, W.; Tanaka, N.; Takaishi, Y. Prenylated xanthones from Hypericum ascyron. J. Nat. Med. 2007, 61, 371-374. [CrossRef]

139. Zhou, Z.B.; Zhang, Y.M.; Luo, J.G.; Kong, L.Y. Cytotoxic polycyclic polyprenylated acylphloroglucinol derivatives and xanthones from Hypericum attenuatum. Phytochem. Lett. 2016, 15, 215-219. [CrossRef]

140. Lou, J.; Wang, H.; Liu, G.Y.; Yang, J.X.; Li, L.M.; Hu, Q.F.; Gao, X.M. Isolation of new xanthone from Hypericum chinense and its cytotoxicity. Asian J. Chem. 2015, 27, 2102-2104. [CrossRef]

141. Matsuoka, E.; Machida, K.; Kikuchi, M. Chemical constituents of Hypericum erectum Thunb. J. Nat. Med. 2008, 62, 467-469. [CrossRef] [PubMed]

142. Wabo, H.; Kowa, T.K.; Lonfouo, A.H.N.; Tchinda, A.T.; Tane, P.; Kikuchi, H.; Frederich, M.; Oshima, Y. Phenolic compounds and terpenoids from Hypericum lanceolatum. Rec. Nat. Prod. 2012, 6, 94-100.

143. Ali, M.; Latif, A.; Zaman, K.; Arfan, M.; Maitland, D.; Ahmad, H.; Ahmad, M. Anti-ulcer xanthones from the roots of Hypericum oblongifolium Wall. Fitoterapia 2014, 95, 258-265. [CrossRef] [PubMed]

144. Ishiguro, K.; Nakajima, M.; Fukumoto, H.; Isoi, K. A xanthone substituted with an irregular monoterpene in cell suspension cultures of Hypericum patulum. Phytochemistry 1995, 39, 903-905. [CrossRef]

145. Sun, L.W.; Sun, Y.N.; Yan, X.T.; Yang, S.Y.; Choi, C.W.; Hyun, J.W.; Kang, H.K.; Paek, K.Y.; Kim, Y.H. Isolation of xanthones from adventitious roots of St. John's Wort (Hypericum perforatum L.) and their antioxidant and cytotoxic activities. Food Sci. Biotechnol. 2013, 22, 945-949. 
146. Xiao, Z.Y.; Shiu, W.K.P.; Zeng, Y.H.; Mu, Q.; Gibbons, S. A naturally occurring inhibitory agent from Hypericum sampsonii with activity against multidrug-resistant staphylococcus aureus. Pharm. Biol. 2008, 46, 250-253. [CrossRef]

147. Tanaka, N.; Takaishi, Y.; Shikishima, Y.; Nakanishi, Y.; Bastow, K.; Lee, K.H.; Takeda, Y.; Kodzhimatov, O.K. Prenylated benzophenones and xanthones from Hypericum scabrum. J. Nat. Prod. 2004, 67, 1870-1875. [CrossRef] [PubMed]

148. Gamiotea-Turro, D.; Cuesta-Rubio, O.; Prieto-Gonzalez, S.; De Simone, F.; Passi, S.; Rastrelli, L. Antioxidative constituents from the leaves of Hypericum styphelioides. J. Nat. Prod. 2004, 67, 869-871. [CrossRef] [PubMed]

149. Al-Khalil, S.; Tosa, H.; Iinuma, M. A xanthone C-glycoside from Iris nigricans. Phytochemistry 1995, 38, 729-731. [CrossRef]

150. Mbouangouere, R.N.; Tane, P.; Ngamga, D.; Khan, S.N.; Choudhary, M.I.; Ngadjui, B.T. A new steroid and $\alpha$-glucosidase inhibitors from Anthocleista schweinfurthii. Res. J. Med. Plant 2007, 1, 106-111.

151. Ateufack, G.; Nguelefack, T.B.; Mbiantcha, M.; Tane, P.; Kamanyi, A. Spasmogenic activity of 1-hydroxy-3,7,8-trimethoxyxanthone isolated from the methanol extract of the stem bark of Anthocleista vogelii planch. (Loganiaceae) in rats. Pharmacologyonline 2007, 3, 374-384.

152. Ee, G.C.; Teo, S.H.; Rahmani, M.; Lim, C.K.; Lim, Y.M.; Go, R. Artomandin, a new xanthone from Artocarpus kemando (Moraceae). Nat. Prod. Res. 2011, 25, 995-1003. [CrossRef] [PubMed]

153. Jayasinghe, U.L.; Samarakoon, T.B.; Kumarihamy, B.M.M.; Hara, N.; Fujimoto, Y. Four new prenylated flavonoids and xanthones from the root bark of Artocarpus nobilis. Fitoterapia 2008, 79, 37-41. [CrossRef] [PubMed]

154. Hashim, N.M.; Rahmani, M.; Sukari, M.A.; Ali, A.M.; Alitheen, N.B.; Go, R.; Ismail, H.B.M. Two new xanthones from Artocarpus obtusus. J. Asian Nat. Prod. Res. 2010, 12, 106-112. [CrossRef] [PubMed]

155. Chen, L.; Zhou, Q.; Li, B.; Liu, S.J.; Dong, J.X. A new flavonoid from Cudrania cochinchinensis. Nat. Prod. Res. 2015, 29, 1217-1221. [CrossRef] [PubMed]

156. Liang, B.; Li, H.R.; Xu, L.Z.; Yang, S.L. Xanthones from the roots of Cudrania fruticosa Wight. J. Asian Nat. Prod. Res. 2007, 9, 393-397. [CrossRef] [PubMed]

157. Quang, T.H.; Ngan, N.T.T.; Yoon, C.S.; Cho, K.H.; Kang, D.G.; Lee, H.S.; Kim, Y.G.; Oh, H. Protein tyrosine phosphatase 1B inhibitors from the roots of Cudrania tricuspidata. Molecules 2015, 20, 11173-11183. [CrossRef] [PubMed]

158. Nakashima, K.; Tanaka, T.; Murata, H.; Kaburagi, K.; Inoue, M. Xanthones from the roots of Maclura cochinchinensis var. gerontogea and their retinoic acid receptor- $\alpha$ agonistic activity. Bioorg. Med. Chem. Lett. 2015, 25, 1998-2001. [PubMed]

159. Ahmad, A.; Singh, D.K.; Fatima, K.; Tandon, S.; Luqman, S. New constituents from the roots of Oenothera biennis and their free radical scavenging and ferric reducing activity. Ind. Crops Prod. 2014, 58, 125-132. [CrossRef]

160. Rezanka, T.; Sigler, K. Hirtusneanoside, an unsymmetrical dimeric tetrahydroxanthone from the Lichen Usnea hirta. J. Nat. Prod. 2007, 70, 1487-1491. [CrossRef] [PubMed]

161. Silveira, E.R.; Falcao, M.J.C.; Kingston, D.G.I.; Glass, T.E. Pentaoxygenated xanthones from Bredemeyera floribunda. Phytochemistry 1995, 39, 1433-1436. [CrossRef]

162. Filho, H.D.S.; Pacheco, L.C.; Andrade, E.S.; Correa, M.J.C.; Araujo, R.N.M.; Guilhon, G.M.S.P.; Da Silva, J.K.R.; Santos, L.S. Xanthones from the roots of Moutabea guianensis Aubl. Molecules 2015, 20, 127-134. [CrossRef] [PubMed]

163. Lin, L.L.; Huang, F.; Chen, S.B.; Yang, D.J.; Chen, S.L.; Yang, J.S.; Xiao, P.G. Xanthones from the roots of Polygala caudata and their antioxidation and vasodilatation activities in vitro. Planta Med. 2005, 71, 372-375. [CrossRef] [PubMed]

164. Deng, S.M.; Yang, X.H.; Zhao, Y.X.; Zhou, J. New xanthones from Polygala crotalarioides. Chem. Res. Chin. Univ. 2006, 22, 400-402. [CrossRef]

165. Klein, L.C.J.; Gandolfi, R.B.; Santin, J.R.; Lemos, M.; Cechinel Filho, V.; Andrade, S.F. Antiulcerogenic activity of extract, fract ions, and some compounds obtained from Polygala cyparissias St. Hillaire \& Moquin (Polygalaceae). Naunyn Schmiedebergs Arch. Pharmacol. 2010, 381, 121-126. [PubMed]

166. Wu, J.F.; Tu, P.F.; Zhan, H.T.; Gao, J.C. Dioxyxanthones from Polygala hongkongensis and their cytotoxicity. Chem. Res. Chin. Univ. 2011, 27, 777-779. 
167. Qing, C.X.; Li, C.J.; Zuo, L.; Yang, J.Z.; Zhang, D.M. Three new xanthones from the roots of Polygala japonica Houtt. J. Asian Nat. Prod. Res. 2009, 11, 465-469.

168. Dao, T.T.; Dang, T.T.; Nguyen, P.H.; Kim, E.; Thuong, P.T.; Won, K. Xanthones from Polygala karensium inhibit neuraminidases from influenza a viruses. Bioorg. Med. Chem. Lett. 2012, 22, 3688-3692. [CrossRef] [PubMed]

169. Jiang, Y.; Zhang, W.; Tu, P.F.; Xu, X.J. Xanthone glycosides from Polygala tenuifolia and their conformational analyses. J. Nat. Prod. 2005, 68, 875-879. [CrossRef] [PubMed]

170. Zhou, Y.H.; Guo, Q.; Jiang, Y.; Tu, P.F. Chemical constituents from the roots of Polygala wattersii Hance. J. Chin. Pharm. Sci. 2014, 23, 723-730. [CrossRef]

171. Yang, X.D.; An, N.; Xu, L.Z.; Yang, S.L. New xanthone glycosides from Securidaca inappendiculata. J. Asian Nat. Prod. Res. 2002, 4, 141-145. [CrossRef] [PubMed]

172. Dibwe, D.F.; Awale, S.; Kadota, S.; Tezuka, Y. Muchimangins A-D: Novel diphenylmethyl-substituted xanthones from Securidaca longepedunculata. Tetrahedron Lett. 2012, 53, 6186-6190. [CrossRef]

173. Pascale, T.; Mondolot, L.; Gargadennec, A.; Kochko, A.; Hamon, S.; Fruchier, A.; Campa, C. First report on mangiferin (C-glucosyl-xanthone) isolated from leaves of a wild coffee plant, Coffea pseudozanguebariae (Rubiaceae). Acta Bot. Gallica 2008, 155, 513-519.

174. Siddiqui, B.S.; Sattar, F.A.; Begum, S.; Gulzar, T.; Ahmad, F. Chemical constituents from the stems of Morinda citrifolia linn. Arch. Pharm. Res. 2007, 30, 793-798. [CrossRef] [PubMed]

175. Tala, M.F.; Wabo, H.K.; Zeng, G.Z.; Ji, C.J.; Tane, P.; Tan, N.H. A prenylated xanthone and antiproliferative compounds from leaves of Pentadesma butyracea. Phytochem. Lett. 2013, 6, 326-330. [CrossRef]

176. Abdissa, N.; Heydenreich, M.; Midiwo, J.O.; Ndakala, A.; Majer, Z.; Neumann, B.; Stammler, H.G.; Sewald, N.; Yenesew, A. A xanthone and a phenylanthraquinone from the roots of Bulbine frutescens, and the revision of six seco-anthraquinones into xanthones. Phytochem. Lett. 2014, 9, 67-73. [CrossRef]

177. Carvalho, M.J.; Carvalho, L.M.; Ferreira, A.M.; Silva, A.M.S. A new xanthone from Hedychium gardnerianum. Nat. Prod. Res. 2003, 17, 445-449. [CrossRef] [PubMed]

178. Li, D.H.; Li, C.X.; Jia, C.C.; Sun, Y.T.; Xue, C.M.; Bai, J.; Hua, H.M.; Liu, X.Q.; Li, Z.L. Xanthones from Garcinia paucinervis with in vitro anti-proliferative activity against HL-60 cells. Arch. Pharm. Res. 2016, 39, 172-177. [CrossRef] [PubMed]

179. Kaennakam, S.; Siripong, P.; Tip-pyang, S. Kaennacowanols A-C, three new xanthones and their cytotoxicity from the roots of Garcinia cowa. Fitoterapia 2015, 102, 171-176. [CrossRef] [PubMed]

180. Sukandar, E.R.; Ersam, T.; Fatmawati, S.; Siripong, P.; Aree, T.; Tip-pyang, S. Cylindroxanthones A-C, three new xanthones and their cytotoxicity from the stem bark of Garcinia cylindrocarpa. Fitoterapia 2016, 108, $62-65$. [CrossRef] [PubMed]

181. Kwon, J.; Hiep, N.T.; Kim, D.W.; Hwang, B.Y.; Lee, H.J.; Mar, W.; Lee, D. Neuroprotective xanthones from the root bark of Cudrania tricuspidata. J. Nat. Prod. 2014, 77, 1893-1901. [CrossRef] [PubMed]

182. Chen, Y.; Fan, H.; Yang, G.Z.; Jiang, Y.; Zhong, F.F.; He, H.W. Prenylated xanthones from the bark of Garcinia xanthochymus and their 1,1-diphenyl-2-picrylhydrazyl (DPPH) radical scavenging activities. Molecules 2010, 15, 7438-7449. [CrossRef] [PubMed]

183. Trisuwan, K.; Ritthiwigrom, T. Benzophenone and xanthone derivatives from the inflorescences of Garcinia cowa. Arch. Pharm. Res. 2012, 35, 1733-1738. [CrossRef] [PubMed]

184. Chen, Y.; He, S.W.; Tang, C.; Li, J.; Yang, G.Z. Caged polyprenylated xanthones from the resin of Garcinia hanburyi. Fitoterapia 2016, 109, 106-112. [CrossRef] [PubMed]

185. Ee, G.C.; Mah, S.H.; Rahmani, M.; Taufiq-Yap, Y.H.; The, S.S.; Yang, M. A new furanoxanthone from the stem bark of Calophyllum inophyllum. J. Asian Nat. Prod. Res. 2011, 13, 956-960. [CrossRef] [PubMed]

186. Gomes, A.S.; Brandão, P.; Fernandes, C.S.G.; da Silva, M.R.P.C.; de Sousa, M.E.D.S.P.; Pinto, M.M.M. Drug-like properties and ADME of xanthone derivatives: The antechamber of clinical trials. Curr. Med. Chem. 2016, 23, 3654-3686. [CrossRef] [PubMed]

187. Han, S.Y.; You, B.H.; Kim, Y.C.; Chin, Y.W.; Choi, Y.H. Dose-independent ADME properties and tentative identification of metabolites of $\alpha$-mangostin from Garcinia mangostana in mice by automated microsampling and UPLC-MS/MS methods. PLoS ONE 2015, 10, e0131587. [CrossRef] [PubMed]

(C) 2017 by the authors. Licensee MDPI, Basel, Switzerland. This article is an open access article distributed under the terms and conditions of the Creative Commons Attribution (CC BY) license (http://creativecommons.org/licenses/by/4.0/). 